\title{
Somatic health care professionals' stigmatization of patients with mental disorder: a scoping review
}

\author{
Ida Nielsen Sølvhøj* (D), Amalie Oxholm Kusier, Pia Vivian Pedersen and Maj Britt Dahl Nielsen
}

\begin{abstract}
Background: Patients with mental disorders have an increased risk of developing somatic disorders, just as they have a higher risk of dying from them. These patients often report feeling devaluated and rejected by health professionals in the somatic health care system, and increasing evidence shows that disparities in health care provision contribute to poor health outcomes. The aim of this review was to map and synthesize literature on somatic health professionals' stigmatization toward patients with mental disorders.
\end{abstract}

Methods: We conducted a scoping review using Arksey and O'Malley's framework and carried out a systematic search in three databases: Cinahl, MEDLINE, and PsycINFO in May-June 2019. Peer-reviewed articles published in English or Scandinavian languages during 2008-2019 were reviewed according to title, abstract and full-text reading. We organized and analyzed data using NVivo.

Results: A total of 137 articles meeting the eligibility criteria were reviewed and categorized as observational studies $(n=73)$ and intervention studies $(n=64)$. A majority of studies $(N=85)$ focused on patients with an unspecified number of mental disorders, while 52 studies focused on specific diagnoses, primarily schizophrenia $(n=13)$, self-harm $(n=13)$, and eating disorders $(n=9)$. Half of the studies focused on health students $(n=64)$, primarily nursing students $(n=26)$ and medical students $(n=25)$, while $(n=66)$ focused on health care professionals, primarily emergency staff $(n=16)$ and general practitioners $(n=13)$. Additionally, seven studies focused on both health professionals and students. A detailed characterization of the identified intervention studies was conducted, resulting in eight main types of interventions.

Conclusions: The large number of studies identified in this review suggests that stigmatizing attitudes and behaviors toward patients with mental disorders is a worldwide challenge within a somatic health care setting. For more targeted interventions, there is a need for further research on underexposed mental diagnoses and knowledge on whether specific health professionals have a more stigmatizing attitude or behavior toward specific mental disorders.

Keywords: Mental health, Mental disorders, Scoping review, Health care professional, Health care student, Stigma

\footnotetext{
* Correspondence: idns@sdu.dk

National Institute of Public Health, University of Southern Denmark,

Studiestræde 6, DK-1455 Copenhagen, Denmark 


\section{Background}

Mental disorders, such as anxiety, depression and substance use disorders, are among the most burdensome disorders in the world $[1,2]$, and the estimated life expectancy is generally $15-20$ years lower for patients with mental disorders compared to the general population [3]. Research shows that somatic disorders are the main cause of this excess mortality [4] and respiratory, digestive and cardiovascular diseases account for the largest impact on mortality among patients with schizophrenia [5]. Not only do patients with mental disorders have a higher risk of developing somatic disorders, they also have a higher risk of dying from them [3, 6-8]. While numerous factors affect the morbidity and mortality of this patient group, increasing evidence shows that disparities in health care provision contribute to poor health outcomes [9-12]. 'Diagnostic overshadowing' the misattribution of physical symptoms to mental illness - is a key concept used in many studies to describe these disparities, as it can contribute to treatment delay and the development of complications [13-15].

In this review, we argue that stigma is key to understanding these disparities. First, stigma can affect multiple life domains and probably has a dramatic bearing on the distribution of life chances in a variety of areas such as earnings, housing and criminal involvement [12]. Second, patients with mental disorders often report feeling devaluated and rejected by health professionals [13, 16], and third, previous research shows that stigma affects patients' willingness to seek treatment and the quality of care [17-20].

Stigma is a complex phenomenon, and definitions vary across disciplines and research fields. According to Link et al. (2001) researchers criticize the term for being too vaguely defined and individually focused. In response to this criticism, Link et al. (2001) proposed a new definition highlighting that: "Stigma exists when elements of labeling, stereotyping, separation, status loss, and discrimination occur together in a power situation that allows them" [12]. This paper focuses specifically on stigma in the somatic health care system toward patients with mental disorders, because suboptimal treatment of serious and potentially life-threatening somatic conditions can have profound negative implications for the patients. Previous literature on mental disorder stigma within somatic health care typically focuses on specific mental disorders or specific health professions and settings [8, 21-24]. Thus, a general overview is lacking. This review aims to provide an overview of the literature on stigmatization among somatic health care professionals toward patients with mental disorders-across different health care professions and mental disorders. More specifically, we aim to: 1) provide an overall characterization of existing observational and intervention studies according to health care profession and diagnosis, 2) provide a detailed characterization of the identified intervention studies, and 3) identify knowledge gaps.

\section{Method}

Scoping reviews aim to create an overview of a research field and to give an indication of the volume of the literature. Furthermore, a scoping review can be helpful in identifying knowledge gaps [25]. The process of conducting a scoping review is systematic and structured [26] and to ensure transparency, we were informed by the PRISMA-ScR guidelines [27] and Arksey and O'Malley's methodological framework [28], which outlines six stages of conducting scoping reviews.

\section{Stage 1: identification of research question}

In health research, it has become increasingly common to engage with stakeholders such as policy makers; clinicians; and patients, just as many research institutions offers research-based collaboration and advice to external parties. These collaborations can help improve study questions and provide more useful findings [29]. In the design of the literature study, we collaborated with The Danish Health Authority and the organization EN AF OS, who aims to destigmatize mental illness in Denmark. They were both involved in developing the research question, and the organization was additionally involved in qualifying keywords and search strings. As recommended by Arksey and O'Malley, we both considered relevant aspects of the research question (e.g. study population, phenomenon of interest and context) and were aware of developing a research question with a wide approach in order to generate breadth of coverage [28].

\section{Stage 2: identifying relevant data}

The first author developed the search strategy in collaboration with research librarians. The search strategy was based on the PICo model that specifies the population (P), phenomenon of interest (I), and context (Co). Following the PICo model the search consisted of two search strings. The first search string specified the population (P), which included patients with frequently occurring mental disorders. We used broad search terms such as 'mental disorders' and additionally performed searches on seven specific disorders, since a too broad search can result in missing relevant studies. The seven specific disorders included: anxiety, depression, bipolar disorder, borderline, schizophrenia, eating disorders, and self-harm. We chose these, because they are among the most common and disabling mental disorders [2]. The second search string specified the phenomenon of interest (I) and context (Co), which included stigmatizing behaviors, attitudes, and perceptions among health professionals, e.g. nurses and physicians, in 
the somatic health care system (see Additional file 1 for full search string). The search was based on keywords (subject headings/ MeSH terms) and free text searches (title, keywords, text). We tested the search string before we formed the final search string. To delimit the search, we applied proximity searching of two words. The search was carried out in May 2019 in three databases: Cinahl, MEDLINE and PsycINFO. Title, abstract and full paper screening were based on inclusion and exclusion criteria. To narrow down the search, we only included studies published 2008-2019 in peer-reviewed journals in English or Scandinavian languages. The temporal delineation was made as we wanted to find the most recent published literature in the field. Protocols, conference literature, book chapters, opinion papers and reviews were excluded. We excluded studies focusing on the mental health of health professionals as well as studies where somatic and psychiatric health professionals could not be separated. While we only included observational studies from the Western Hemisphere, we applied no geographical exclusion criteria for the intervention studies. This is because we wanted to gain insight into experiences from interventions conducted all over the world and because the wide range of interventions may contribute as inspiration for future prevention work. We made the choice of only including observational studies from the Western Hemisphere in collaboration with the organization EN AF OS, to narrow down the search.

\section{Stage 3: data selection}

We stored the studies in Endnote and removed duplicates. Next, we moved the studies to the review manager Covidence and performed another duplicate check, after which we began the screening process of the 11,798 identified studies. During the initial title screening, we excluded 10,622 studies for being out of scope. Additionally, we excluded 928 studies during abstract screening for not meeting the inclusion criteria. Of the remaining 248 studies, 111 studies were excluded for not meeting the inclusion criteria.

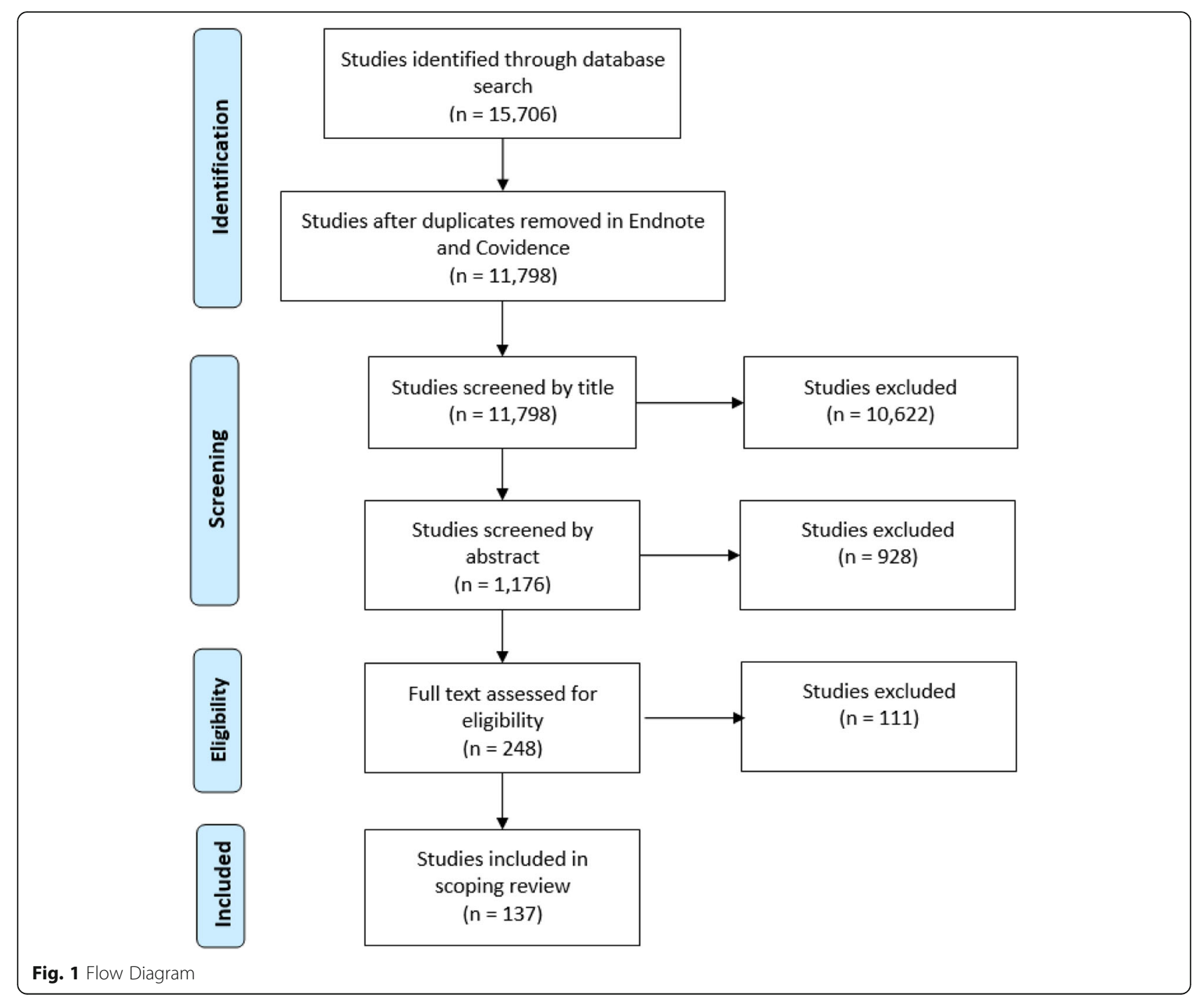


Finally, we included a total of 137 studies in the scoping review. Figure 1 summarizes the literature search and study selection.

\section{Stage 4 and 5: charting and collating data}

We used NVivo to organize and analyze the data. The data charting took the following information into consideration: author(s), year of publication, country of origin, study design, study population (health professionals in the somatic health system), sample size, and mental disorders. Additionally, we coded the scope, method, intervention type, instrument, and outcome in each study. Subsequently, we summarized the studies by study design: observational studies and intervention studies.

\section{Stage 6: consultation exercise}

As briefly described above, we collaborated with The Danish Health Authority and the organization EN AF OS, in the development of the design of the literature review and of the research question. The organization was additionally involved in the process of further qualifying keywords and search strings to ensure that relevant keywords, including mental disorders and specific health professionals were not omitted. Thus, the organization played an important role in quality assuring the first two stages of the scoping review. Further, we discussed the findings of the scoping review with the organization and learned that our findings corresponded well with the organization's knowledge of the phenomenon.

\section{Results}

In total, we included 137 studies of which 73 were observational studies and 64 were intervention studies. In the following, we describe and categorize the identified studies according to diagnosis and health care profession. All included studies examined stigmatizing behaviors, attitudes, and/or perceptions among health professionals in the somatic health care system toward patients with mental disorders. For the sake of readability, we will primarily refer to this information as 'attitudes and behaviors'.

\section{Categorization of health professionals and patients}

To create an overview of the wide range of different health professions included in the identified studies, we divided the health professionals into 13 categories (Fig. 2). Some studies focused on students rather than trained professionals, and we categorized these studies separately. Other studies included both students and professionals. Moreover, while some studies included well-defined groups of health professionals, such as nurses or general practitioners, others did not focus on specific health professionals. These studies were categorized under the heading 'Health professionals', e.g. various hospital employees. Similarly, the category 'Health care students' refers to various students within health education. However, we also identified studies examining both students and health professionals, which formed the broad category 'Health professionals and health care students'. Finally, the category 'Medical doctors' covers all other types of medical doctors besides general practitioners, e.g. surgeons or different types of medical specialists.

We also categorized the identified studies according to the patients' diagnoses and formed seven categories. Five of the categories cover specific diagnoses. In addition, we included a 'Mixed mental disorders' category. This category includes studies not confined to a specific mental disorder or studies that examined multiple diagnoses. Furthermore, the category 'Dual diagnosis' covers studies examining patients with a mental disorder and a substance use disorder.

\section{Characteristics of the observational studies}

The main purpose of the observational studies was to investigate the magnitude of stigmatizing attitudes

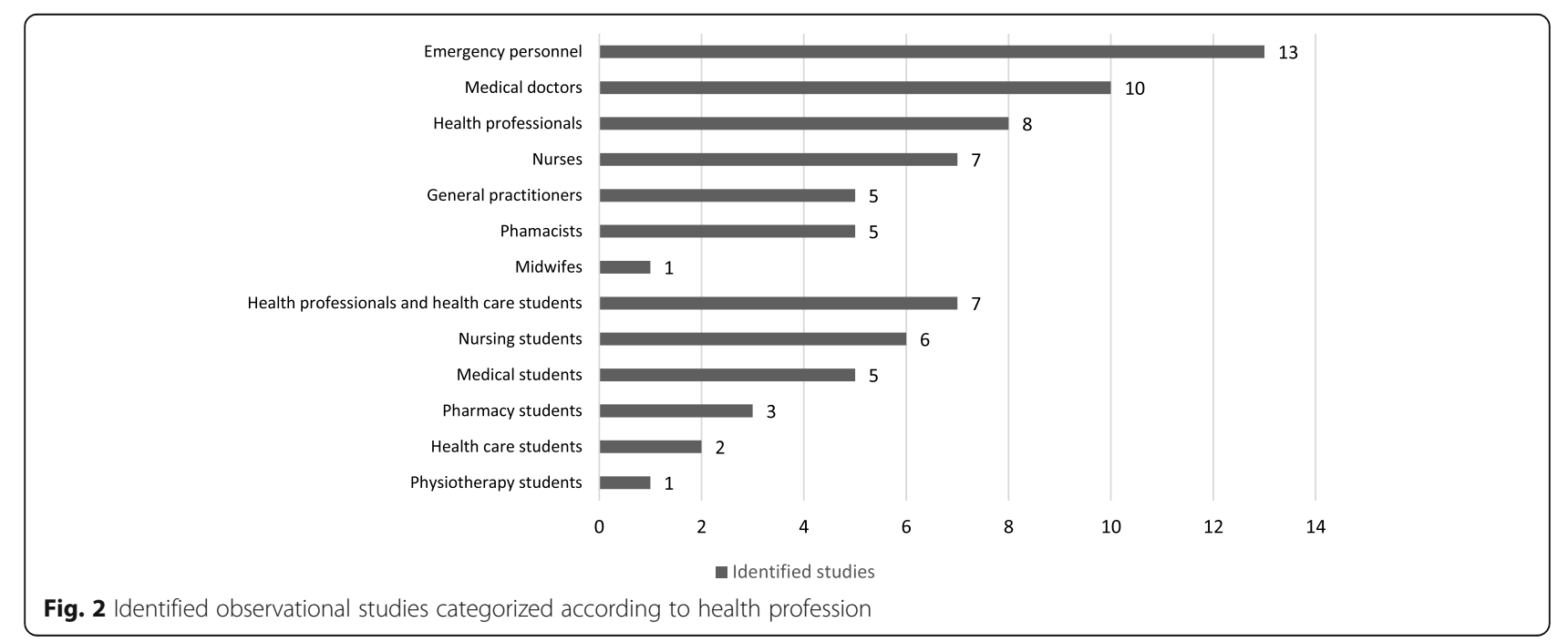


and behaviors among health professionals in the somatic health care system toward patients with mental disorders. In total, we identified 73 observational studies, all from the Western Hemisphere. About half of the studies were from Europe $(n=41)$, including a large proportion of studies from England $(n=16)$, followed by North America $(n=15)$, Oceania $(n=11)$, and Asia $(n=3)$. Furthermore, we identified three studies comparing populations across countries. Most studies used quantitative methods $(n=58)$; however, we also identified qualitative studies $(n=13)$ and mixed methods studies $(n=2)$.

As illustrated in Fig. 2, we found that most studies $(n=13)$ focused on emergency personnel, followed by medical doctors $(n=10)$ and health professionals $(n=8)$. Most studies $(n=40)$ focused on several, different diagnoses (Table 1). In studies focusing on single, specific diagnoses, the most frequent mental disorders were selfharm $(\mathrm{n}=10)$ and schizophrenia $(n=9)$.

We combined type of health profession with diagnosis as shown in Table 1 . The table shows that a relatively large proportion of studies examined attitudes and behaviors among emergency staff toward patients who selfharm $(n=6)$. In contrast, studies examining attitudes and behaviors among medical doctors $(n=10)$ were divided into a wide range of diagnoses, such as depression $(\mathrm{n}=1)$, schizophrenia $(\mathrm{n}=1)$, eating disorders $(n=2)$, self-harm $(\mathrm{n}=2)$, mixed mental disorders $(n=3)$, and dual diagnosis $(\mathrm{n}=1)$.

For a detailed description of study design, target group, sample size and diagnosis on observational studies, see Table 2.

\section{Characteristics of the intervention studies}

The main purpose of the intervention studies was to evaluate interventions to reduce health professionals' stigmatizing attitudes and behaviors toward people with mental disorders. We identified 64 intervention studies, most of which were from North America $(n=19)$, followed by studies from Europe $(n=16)$, Oceania $(n=$ $13)$, Asia $(n=13)$, and Africa $(n=1)$. In addition, two interventions studies were comparative studies. Most studies were based on quantitative methods $(n=51)$; seven were mixed methods studies, and six were qualitative studies.

Most intervention studies focused on changing students' attitudes and behaviors toward patients with mental disorders; 20 of these studies focused on medical students, 20 focused on nursing students and six focused on pharmacy students, as illustrated in Fig. 3. Furthermore, eight studies focused on general practitioners, constituting the third-largest category when distributing the studies by health profession.
Table 1 Combination of diagnoses and health care profession (observational studies)

\begin{tabular}{|c|c|c|}
\hline Diagnosis & Health care profession & $\begin{array}{l}\text { Number of } \\
\text { studies }\end{array}$ \\
\hline \multirow[t]{3}{*}{ Depression $(n=3)$} & Health care students & 1 \\
\hline & Pharmacists & 1 \\
\hline & Medical doctors & 1 \\
\hline \multirow[t]{2}{*}{ Borderline $(n=2)$} & Nurses & 1 \\
\hline & Emergency personnel & 1 \\
\hline \multirow[t]{7}{*}{ Schizophrenia $(n=9)$} & $\begin{array}{l}\text { Health professionals and health } \\
\text { care students }\end{array}$ & 2 \\
\hline & Nursing students & 1 \\
\hline & Pharmacy students & 2 \\
\hline & Pharmacists & 1 \\
\hline & Medical students & 1 \\
\hline & Medical doctors & 1 \\
\hline & General practitioners & 1 \\
\hline \multirow[t]{4}{*}{ Eating disorder $(n=5)$} & Health care students & 1 \\
\hline & Health professionals & 1 \\
\hline & Medical doctors & 2 \\
\hline & General practitioners & 1 \\
\hline \multirow[t]{3}{*}{ Self-harm $(n=10)$} & Health professionals & 2 \\
\hline & Medical doctors & 2 \\
\hline & Emergency personnel & 6 \\
\hline \multirow{12}{*}{$\begin{array}{l}\text { Mixed mental } \\
\text { disorders }(n=40)\end{array}$} & Physiotherapy students & 1 \\
\hline & Midwifes & 1 \\
\hline & $\begin{array}{l}\text { Health professionals and health } \\
\text { care students }\end{array}$ & 5 \\
\hline & Nursing students & 6 \\
\hline & Nurses & 5 \\
\hline & Health professionals & 3 \\
\hline & Pharmacy students & 1 \\
\hline & Pharmacists & 4 \\
\hline & Medical students & 4 \\
\hline & Medical doctors & 3 \\
\hline & General practitioners & 3 \\
\hline & Emergency personnel & 5 \\
\hline \multirow[t]{3}{*}{ Dual diagnosis $(n=4)$} & Health professionals & 2 \\
\hline & Medical doctors & 1 \\
\hline & Emergency personnel & 1 \\
\hline Total & & 73 \\
\hline
\end{tabular}

Most intervention studies $(n=45)$ did not focus on patients with a specific mental disorder, but typically on attitudes and behaviors toward multiple mental disorders or mental disorder in general as shown in Table 2 . Depression $(n=5)$, eating disorder $(n=4)$, and schizophrenia $(n=4)$ were among the most common diagnoses. 
Table 2 Observational studies

\begin{tabular}{|c|c|c|c|c|c|}
\hline $\begin{array}{l}\text { First author, year, } \\
\text { reference }\end{array}$ & Country & Population & $\begin{array}{l}\text { Sample } \\
\text { size }\end{array}$ & Diagnosis & Design \\
\hline Abood, 2009 [30] & UK & Medical doctors & $N=47$ & Self-harm & Quantitative \\
\hline Anderson, 2017 [31] & USA & Medical doctors & $N=80$ & Eating disorder & Quantitative \\
\hline Arbanas, 2019 [32] & Croatia & Health professionals & $N=387$ & Mixed & Quantitative \\
\hline Artis, 2013 [33] & UK & Emergency personnel & $N=10$ & Self-harm & Qualitative \\
\hline Arvaniti, 2009 [34] & Greece & $\begin{array}{l}\text { Health professionals and health } \\
\text { care students }\end{array}$ & $N=592$ & Mixed & Quantitative \\
\hline Avery, 2019 [35] & USA & Medical doctors & $N=411$ & Dual diagnosis & Quantitative \\
\hline Bannatyne, 2017 [36] & Australia & Health care students & $N=126$ & Eating disorder & Quantitative \\
\hline Bell, 2010 [37] & $\begin{array}{l}\text { Australia, Belgium, India, Finland, } \\
\text { Estonia, Latvia }\end{array}$ & Pharmacy students & $N=649$ & Schizophrenia & Quantitative \\
\hline Bell, 2008 [38] & $\begin{array}{l}\text { Australia, Belgium, India, Finland, } \\
\text { Estonia, Latvia }\end{array}$ & Pharmacy students & $N=642$ & Mixed & Quantitative \\
\hline Bjorkman, 2008 [39] & Sweden & Nurses & $N=120$ & Mixed & Quantitative \\
\hline Brunero, 2017 [40] & Australia & Nurses & $N=16$ & Mixed & Qualitative \\
\hline Castillejos, 2019 [41] & Spain & General practitioners & $N=145$ & Mixed & Quantitative \\
\hline Ceylan, 2019 [42] & Turkey & Nurses & $N=186$ & Schizophrenia & Quantitative \\
\hline Chapman, 2014 [43] & Australia & Emergency Personnel & $N=186$ & Self-harm & Quantitative \\
\hline Clifton, 2016 [44] & UK & Health professionals & $N=85$ & Mixed & Qualitative \\
\hline Conlon, 2012 [45] & Ireland & Emergency personnel & $N=87$ & Self-harm & Quantitative \\
\hline Crapanzano, 2018 [46] & USA & Medical doctors & $N=96$ & Depression & Quantitative \\
\hline Currin, 2009 [47] & UK & General practitioners & $N=154$ & Eating disorders & Quantitative \\
\hline Cutler, 2009 [48] & USA & Medical students & $N=47$ & Mixed & Qualitative \\
\hline Dixon, 2008 [49] & UK & Medical students & $N=1081$ & Mixed & Quantitative \\
\hline Ewalds-Kvist, 2013 [50] & Sweden & Nursing students & $N=246$ & Mixed & Quantitative \\
\hline Gawley, 2011 [51] & Canada & Health care students & $N=309$ & Depression & Quantitative \\
\hline Giannetti, 2018 [52] & USA & Pharmacists & $N=239$ & Mixed & Quantitative \\
\hline Gordon, 2012 [53] & UK & Emergency personnel & $N=32$ & Mixed & Quantitative \\
\hline $\begin{array}{l}\text { Granados-Gamez, } 2017 \\
\text { [54] }\end{array}$ & Spain & Nursing students & $N=194$ & Mixed & Quantitative \\
\hline Happell, 2008 [55] & Australia & Nursing students & $N=148$ & Mixed & Quantitative \\
\hline Happell, 2018 [56] & $\begin{array}{l}\text { Australia, Ireland, Finland, Norway, } \\
\text { Netherland }\end{array}$ & Nursing students & $N=423$ & Mixed & Quantitative \\
\hline $\begin{array}{l}\text { Heyward-Chaplin, } 2018 \\
\text { [57] }\end{array}$ & UK & Health professionals & $N=59$ & Self-harm & Quantitative \\
\hline $\begin{array}{l}\text { Ihalainen-Tamlander, } \\
2016 \text { [58] }\end{array}$ & Finland & Nurses & $N=218$ & Mixed & Quantitative \\
\hline Janouskova, 2017 [59] & The Czech Republic & $\begin{array}{l}\text { Health professionals and health } \\
\text { care students }\end{array}$ & $N=308$ & Mixed & Quantitative \\
\hline Jones, 2009 [60] & USA & Medical doctors & $N=51$ & Mixed & Quantitative \\
\hline Koning, 2018 [61] & Australia & Emergency personnel & $N=15$ & Self-harm & Qualitative \\
\hline Thongpriwan, 2015 [62] & USA & Nursing students & $N=229$ & Mixed & Quantitative \\
\hline Kopera, 2015 [63] & Polonia & $\begin{array}{l}\text { Health professionals and health } \\
\text { care students }\end{array}$ & $N=57$ & Mixed & Quantitative \\
\hline Korszun, 2012 [64] & UK & Medical students & $N=760$ & Mixed & Quantitative \\
\hline Kuzman, 2014 [65] & The Czech Republic & Medical students & $N=199$ & Mixed & Quantitative \\
\hline Leddy, 2009 [66] & USA & Medical doctors & $N=504$ & Eating disorder & Quantitative \\
\hline Liekens, 2012 [67] & Belgium & Pharmacists & $N=149$ & Depression & Quantitative \\
\hline
\end{tabular}


Table 2 Observational studies (Continued)

\begin{tabular}{|c|c|c|c|c|c|}
\hline $\begin{array}{l}\text { First author, year, } \\
\text { reference }\end{array}$ & Country & Population & $\begin{array}{l}\text { Sample } \\
\text { size }\end{array}$ & Diagnosis & Design \\
\hline Magliano, 2011 [68] & Italy & Medical students & $N=194$ & Schizophrenia & Quantitative \\
\hline Magliano, 2017 [69] & Italy & General practitioners & $N=387$ & Schizophrenia & Quantitative \\
\hline McCann, 2018 [70] & Australia & Emergency personnel & $N=1230$ & Dual diagnosis & Quantitative \\
\hline McCarthy, 2010 [71] & Ireland & Emergency Personnel & $N=68$ & Self-harm & Quantitative \\
\hline Morral, 2016 [72] & UK & Pharmacists & $N=351$ & Mixed & Quantitative \\
\hline $\begin{array}{l}\text { Muehlenkamp, } 2013 \\
\text { [73] }\end{array}$ & Belgium & Health professionals & $N=342$ & Self-harm & Quantitative \\
\hline Nash, 2013 [13] & UK & Emergency personnel & $N=39$ & Mixed & Qualitative \\
\hline Nauta, 2019 [74] & Netherlands & Medical doctors & $N=187$ & Mixed & Quantitative \\
\hline Neauport, 2012 [75] & France & Medical doctors & $N=322$ & Mixed & Quantitative \\
\hline Noonan, 2018 [76] & Ireland & Midwifes & $N=157$ & Mixed & Quantitative \\
\hline Nutt, 2017 [77] & Scotland & Health professionals & $N=113$ & Dual diagnosis & Quantitative \\
\hline O'Reilly, 2012 [78] & Australia & $\begin{array}{l}\text { Health professionals and health } \\
\text { care students }\end{array}$ & $N=23$ & Mixed & Qualitative \\
\hline O'Reilly, 2015 [79] & Australia & Pharmacists & $N=188$ & Schizophrenia & Quantitative \\
\hline Peitl, 2011 [80] & Croatia & $\begin{array}{l}\text { Health professionals and health } \\
\text { care students }\end{array}$ & $N=151$ & Mixed & Quantitative \\
\hline Perboell, 2015 [81] & Denmark & Emergency personnel & $N=122$ & Self-harm & Quantitative \\
\hline Prener, 2015 [82] & USA & Emergency personnel & $N=20$ & Mixed & Qualitative \\
\hline Rai, 2019 [83] & UK & Medical doctors & $N=37$ & Self-harm & $\begin{array}{l}\text { Mixed } \\
\text { methods }\end{array}$ \\
\hline Rao, 2009 [84] & UK & Health professionals & $N=108$ & Dual diagnosis & Quantitative \\
\hline Raveneau, 2014 [85] & USA & Health professionals & $N=82$ & Eating disorder & Quantitative \\
\hline Reavley, 2014 [86] & Australia & Health professionals & $N=1536$ & Mixed & Quantitative \\
\hline Rickles, 2010 [87] & USA & Pharmacists & $N=292$ & Mixed & Quantitative \\
\hline Sandhu, 2019 [88] & Canada & $\begin{array}{l}\text { Health professionals and health } \\
\text { care students }\end{array}$ & $N=538$ & Schizophrenia & Quantitative \\
\hline Schafer, 2011 [89] & UK & Nursing students & $N=288$ & Mixed & Quantitative \\
\hline Schmidt, 2017 [90] & Netherlands & General practitioners & $N=63$ & Mixed & Quantitative \\
\hline Serafini, 2011 [91] & Italy & $\begin{array}{l}\text { Health professionals and health } \\
\text { care students }\end{array}$ & $N=202$ & Schizophrenia & Quantitative \\
\hline Shefer, 2014 [15] & UK & Emergency personnel & $N=39$ & Mixed & Qualitative \\
\hline Stumbo, 2018 [92] & USA & General practitioners & $N=597$ & Mixed & $\begin{array}{l}\text { Mixed } \\
\text { methods }\end{array}$ \\
\hline Treloar, 2009 [93] & Australia & Emergency personnel & $N=140$ & $\begin{array}{l}\text { Borderline personality } \\
\text { disorder }\end{array}$ & Qualitative \\
\hline $\begin{array}{l}\text { Van Nieuwenhui, } 2013 \\
\text { [94] }\end{array}$ & UK & Emergency personnel & $N=25$ & Mixed & Qualitative \\
\hline Volmer, 2008 [95] & Estonia & Pharmacy students & $N=157$ & Schizophrenia & Quantitative \\
\hline Weare, 2019 [96] & Australia & Nurses & $N=40$ & Mixed & Quantitative \\
\hline Winkler, 2016 [97] & The Czech Republic & Medical doctors & $N=3010$ & Mixed & Quantitative \\
\hline Woollaston, 2008 [98] & UK & Nurses & $N=6$ & $\begin{array}{l}\text { Borderline personality } \\
\text { disorder }\end{array}$ & Qualitative \\
\hline Yildirim, 2015 [99] & Turkey & Physiotherapy students & $N=524$ & Mixed & Quantitative \\
\hline Zolnierek, 2012 [100] & USA & Nurses & $N=1$ & Mixed & Qualitative \\
\hline
\end{tabular}




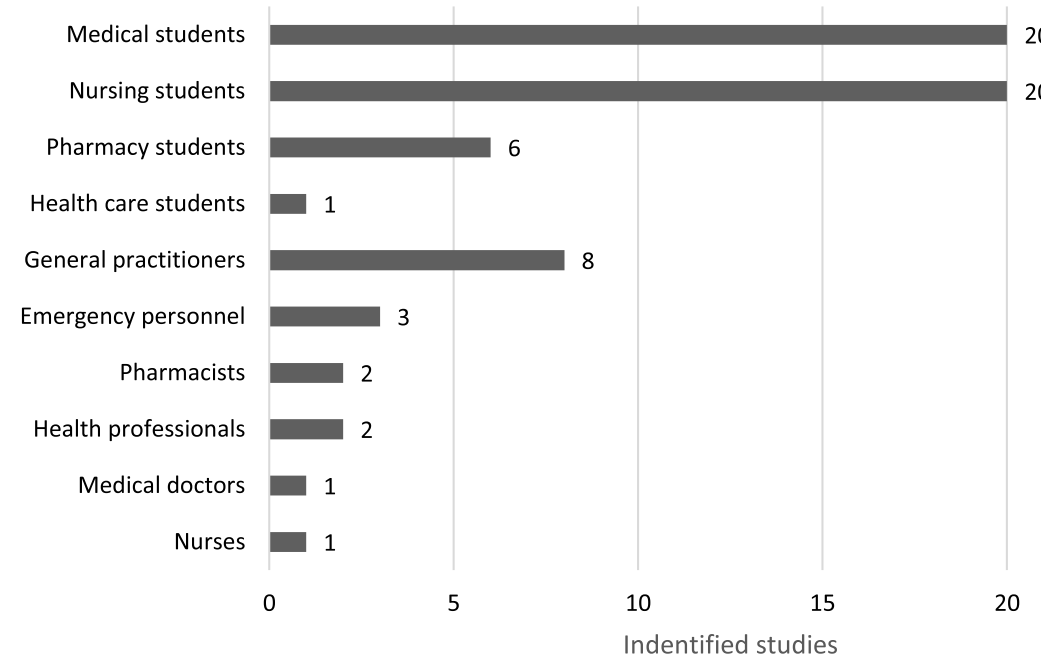

Fig. 3 Identified intervention studies categorized according to health profession

By combining health profession with diagnosis (Table 3), we found that most of the studies focusing on attitudes and behaviors among nursing students did not focus on a specific mental disorder, as 17 of the 20 identified studies looked at multiple diagnoses or mental disorder in general. Similarly, 16 of 20 identified studies examining attitudes and behaviors among medical students focused on multiple diagnoses or mental disorder in general. We found a similar pattern for studies examining attitudes and behaviors among pharmacy students $(n=6)$ and health care students $(n=1)$.

For a detailed description of study design, target group, sample size, and diagnosis in the intervention studies, see Table 4.

Table 3 Combination of diagnoses and health care profession (intervention studies)

\begin{tabular}{lll}
\hline Diagnosis & Health care profession & Number of studies \\
\hline Depression $(n=5)$ & General practitioners & 3 \\
& Pharmacists & 2 \\
Borderline $(n=2)$ & Emergency personnel & 1 \\
Schizophrenia $(n=4)$ & Health professionals & 1 \\
& Medical doctors & 1 \\
Eating disorder $(n=4)$ & Medical students & 3 \\
& General practitioners & 1 \\
Self-harm $(n=3)$ & Medical students & 1 \\
& Nursing students & 2 \\
Bipolar disorder $(n=1)$ & Emergency personnel & 2 \\
Mixed mental disorders $(n=45)$ & Nursing students & 1 \\
& Health professionals & 1 \\
Total & General practitioners & 4 \\
\hline
\end{tabular}




\section{Intervention types and content}

Of the 64 included intervention studies, 47 targeted health care students while 17 targeted health professionals. To provide a more detailed characterization of the type and content of the identified intervention studies, we categorized the interventions into eight main types: four targeting students and four targeting health professionals. We categorized the interventions targeting students based on the content of the intervention, whereas we categorized interventions targeting health professionals according to the content and specific health profession (e.g. nurses or medical doctors) because these were often closely related. We present one example of each intervention type, focusing on examples that are illustrative of the intervention types, welldescribed in the articles, and show a geographical breadth and variation between health care students and professionals. For a detailed description of the intervention studies, see Table 4.

\section{Interventions targeting students}

We identified 47 intervention studies targeting health care students. We categorized these into four different types of interventions: a) Lectures b) Expeditionary interventions c) Contact-based interventions, and d) Observational studies of the effect of attending medical school. Many interventions included a mix of different activities. We divided the interventions according to the most prominent ones. The interventions most often targeted nursing students $(n=20)$ or medical students $(n=$ 20), while six interventions targeted pharmacy students and one targeted a mixed group of students.

\section{Lectures}

Interventions based on lectures $(n=13)$ were characterized by a teacher-centered approach, and typically took place in a classroom where the teacher provided different educational programs [101, 103, 105, 112, 115-117, $126,128,148,152,155,158]$. The topics of the lectures varied, including e.g. doctor-patient relationships [101], empathy [103], mental health literacy [152], social distancing [155], fear [148], and knowledge about how patients with mental disorders experience encounters with the somatic health care system [158]. In an example of a classical teaching intervention from Australia, pharmacy students participated in two 12-h Mental Health First Aid courses. The classes addressed themes such as symptoms, evidence-based treatment of several mental disorders, early warning signs of mental disorder, and how to provide initial help to people in a mental health crisis. The courses involved, e.g., case studies and group activities [152].

\section{Expeditionary interventions}

These interventions $(n=17)$ had in common that they primarily took place outside of the classroom and included clerkships and field trips, e.g. to psychiatric wards $[102,106,107,118-120,129,131,139,141,147,149$, $153,156,157,159]$. The interventions lasted from four hours a week for three weeks [106] to full time for eight weeks [139], and some also included lectures on mental health and psychiatry $[106,118,119,139,157]$. While all 17 interventions aimed to reduce stigma, some also investigated the impact on a) students' interest in psychiatry, b) psychiatry as a career choice, and c) attitudes toward psychiatry.

In some interventions, students visited psychiatric facilities [131] or pharmacies [115], since a visit at local pharmacies allowed pharmacy students to meet patients with mental disorders. Other interventions were mental health camps consisting of a 2-5-day immersive learning program outside of the 'typical' clinical setting, where students could meet and interact with people with a mental disorder at camp sites [147, 159]. One of these interventions included students in the United States, who participated in a mental health camp after receiving didactic teaching. The camp consisted of two days working with a group of patients from the local mental health service. The program included trust and confidencebuilding exercises and socialization through joint preparation of meals and leisure activities. Following the camp, students attended a 15-week mental health placement at either a community facility or a hospital [159].

\section{Contact-based interventions}

Contact-based interventions $(n=12)$ had in common that they focused on facilitated encounters with patients with mental disorders $[110,123,125,127,130,132,133$, $142,146,151,154,164]$. These types of interventions were mainly characterized by patients with mental disorders being involved in the lectures, either as educators $[130,151]$ or as visitors giving testimonies [133, 142, 154]. In some cases, the testimonies were introduced to students via video display $[110,123,132,164]$. In contrast to interventions based on expeditionary learning, contact-based interventions typically took place in classrooms or other educational settings.

To exemplify, in Spain nursing students participated in a 90-min intervention including testimonies from a mental health professional, a person with a mental disorder and a family member of another person with a mental disorder. They described their experiences with mental disorder, e.g., how the disorder emerged, symptoms and side effects of medication, problems related to family coexistence, and problems in the workplace. Following this, a 30-min discussion among students and the presenters was held [142]. 
Table 4 Intervention studies

\begin{tabular}{|c|c|c|c|c|c|c|}
\hline First author, year & Country & Population & $\begin{array}{l}\text { Sample } \\
\text { size }\end{array}$ & Diagnosis & Design & Intervention \\
\hline Airagnes, 2014 [101] & France & $\begin{array}{l}\text { Medical } \\
\text { students }\end{array}$ & $N=163$ & Mixed & $\begin{array}{l}\text { Quasi-experimental } \\
\text { with control group }\end{array}$ & Lectures \\
\hline Arbanas, 2018 [102] & Croatia & $\begin{array}{l}\text { Nursing } \\
\text { students }\end{array}$ & $N=51$ & Mixed & $\begin{array}{l}\text { Quasi-experimental } \\
\text { without control group }\end{array}$ & Expeditionary interventions \\
\hline $\begin{array}{l}\text { Bannatyne, } 2015 \\
\text { [103] }\end{array}$ & Australia & $\begin{array}{l}\text { Medical } \\
\text { students }\end{array}$ & $N=41$ & Eating disorder & $\begin{array}{l}\text { Quasi-experimental } \\
\text { with control group }\end{array}$ & Lectures \\
\hline Beaulieu, 2017 [104] & Canada & $\begin{array}{l}\text { General } \\
\text { practitioners }\end{array}$ & $N=73$ & Mixed & $\mathrm{RCT}$ & $\begin{array}{l}\text { Interventions targeting general } \\
\text { practitioners and medical doctors }\end{array}$ \\
\hline Bilge, 2017 [105] & Turkey & $\begin{array}{l}\text { Nursing } \\
\text { students }\end{array}$ & $N=322$ & Mixed & Other & Lectures \\
\hline $\begin{array}{l}\text { Bingham, } 2018 \\
\text { [106] }\end{array}$ & New Zealand & $\begin{array}{l}\text { Nursing } \\
\text { students }\end{array}$ & $N=45$ & Mixed & $\begin{array}{l}\text { Quasi-experimental } \\
\text { without control group }\end{array}$ & Expeditionary interventions \\
\hline Brenner, 2011 [107] & USA & $\begin{array}{l}\text { Medical } \\
\text { students }\end{array}$ & $N=100$ & Mixed & Qualitative & Expeditionary interventions \\
\hline $\begin{array}{l}\text { Calloway, } 2017 \\
\text { [108] }\end{array}$ & USA & Nurses & $N=82$ & Mixed & Qualitative & $\begin{array}{l}\text { Interventions targeting health } \\
\text { professionals }\end{array}$ \\
\hline Chiles, 2017 [109] & USA & $\begin{array}{l}\text { Medical } \\
\text { students }\end{array}$ & $N=289$ & Mixed & $\begin{array}{l}\text { Quasi-experimental } \\
\text { without control group }\end{array}$ & Observational studies \\
\hline Clement, 2012 [110] & UK & $\begin{array}{l}\text { Nursing } \\
\text { students }\end{array}$ & $N=216$ & Mixed & $\mathrm{RCT}$ & Contact-based interventions \\
\hline $\begin{array}{l}\text { Coppens, } 2018 \\
\text { [111] }\end{array}$ & $\begin{array}{l}\text { Portugal, Germany, } \\
\text { Ireland, Hungary }\end{array}$ & $\begin{array}{l}\text { General } \\
\text { practitioners }\end{array}$ & $N=208$ & Depression & $\begin{array}{l}\text { Quasi-experimental } \\
\text { without control group }\end{array}$ & $\begin{array}{l}\text { Interventions targeting general } \\
\text { practitioners and medical doctors }\end{array}$ \\
\hline Crisafulli, 2008 [112] & USA & $\begin{array}{l}\text { Nursing } \\
\text { students }\end{array}$ & $N=115$ & Eating disorder & $\begin{array}{l}\text { Quasi-experimental } \\
\text { with control group }\end{array}$ & Lectures \\
\hline Crockett, 2009 [113] & Australia & Pharmacists & $N=32$ & Depression & $\mathrm{RCT}$ & Interventions targeting pharmacists \\
\hline $\begin{array}{l}\text { Demiroren, } 2016 \\
\text { [114] }\end{array}$ & Turkey & $\begin{array}{l}\text { Medical } \\
\text { students }\end{array}$ & $N=190$ & Mixed & $\begin{array}{l}\text { Quasi-experimental } \\
\text { with control group }\end{array}$ & Expeditionary interventions \\
\hline Dipaula, 2011 [115] & USA & $\begin{array}{l}\text { Pharmacy } \\
\text { students }\end{array}$ & $N=278$ & Mixed & $\begin{array}{l}\text { Quasi-experimental } \\
\text { with control group }\end{array}$ & Lectures \\
\hline Duffy, 2016 [116] & USA & $\begin{array}{l}\text { Nursing } \\
\text { students }\end{array}$ & $N=131$ & Eating disorder & $\begin{array}{l}\text { Quasi-experimental } \\
\text { without control group }\end{array}$ & Lectures \\
\hline Duman, 2017 [117] & Turkey & $\begin{array}{l}\text { Nursing } \\
\text { students }\end{array}$ & $N=202$ & Mixed & $\begin{array}{l}\text { Quasi-experimental } \\
\text { with control group }\end{array}$ & Lectures \\
\hline $\begin{array}{l}\text { Economou, } 2017 \\
{[118]}\end{array}$ & Greece & $\begin{array}{l}\text { Medical } \\
\text { students }\end{array}$ & $N=678$ & Mixed & $\begin{array}{l}\text { Quasi-experimental } \\
\text { without control group }\end{array}$ & Expeditionary interventions \\
\hline $\begin{array}{l}\text { Economou, } 2012 \\
\text { [119] }\end{array}$ & Greece & $\begin{array}{l}\text { Medical } \\
\text { students }\end{array}$ & $N=158$ & Schizophrenia & $\begin{array}{l}\text { Quasi-experimental } \\
\text { without control group }\end{array}$ & Expeditionary interventions \\
\hline Eksteen, 2017 [120] & $\begin{array}{l}\text { The South African } \\
\text { Republic }\end{array}$ & $\begin{array}{l}\text { Medical } \\
\text { students }\end{array}$ & $N=616$ & Mixed & $\begin{array}{l}\text { Quasi-experimental } \\
\text { without control group }\end{array}$ & Expeditionary interventions \\
\hline $\begin{array}{l}\text { Esen Danaci, } 2016 \\
\text { [121] }\end{array}$ & Turkey & $\begin{array}{l}\text { Medical } \\
\text { students }\end{array}$ & $N=106$ & Schizophrenia & $\begin{array}{l}\text { Quasi-experimental } \\
\text { without control group }\end{array}$ & Observational studies \\
\hline Failde, 2014 [122] & Spain & $\begin{array}{l}\text { Medical } \\
\text { students }\end{array}$ & $N=171$ & Mixed & $\begin{array}{l}\text { Quasi-experimental } \\
\text { without control group }\end{array}$ & Observational studies \\
\hline $\begin{array}{l}\text { Fernandez, } 2016 \\
\text { [123] }\end{array}$ & Malaysia & $\begin{array}{l}\text { Medical } \\
\text { students }\end{array}$ & $N=102$ & Mixed & $\mathrm{RCT}$ & Contact-based interventions \\
\hline $\begin{array}{l}\text { Flanagan, } 2016 \\
\text { [124] }\end{array}$ & USA & $\begin{array}{l}\text { General } \\
\text { practitioners }\end{array}$ & $N=27$ & Mixed & $\begin{array}{l}\text { Quasi-experimental } \\
\text { with control group }\end{array}$ & $\begin{array}{l}\text { Interventions targeting general } \\
\text { practitioners and medical doctors }\end{array}$ \\
\hline Fokuo, 2017 [125] & USA & $\begin{array}{l}\text { Nursing } \\
\text { students }\end{array}$ & $N=70$ & Mixed & Qualitative & Contact-based interventions \\
\hline Gable, 2011 [126] & USA & $\begin{array}{l}\text { Pharmacy } \\
\text { students }\end{array}$ & $N=39$ & Mixed & $\begin{array}{l}\text { Quasi-experimental } \\
\text { with control group }\end{array}$ & Lectures \\
\hline Galletly, 2011 [127] & Australia & $\begin{array}{l}\text { Medical } \\
\text { students }\end{array}$ & $N=87$ & Schizophrenia & $\begin{array}{l}\text { Quasi-experimental } \\
\text { without control group }\end{array}$ & Contact-based interventions \\
\hline
\end{tabular}


Table 4 Intervention studies (Continued)

\begin{tabular}{|c|c|c|c|c|c|c|}
\hline First author, year & Country & Population & $\begin{array}{l}\text { Sample } \\
\text { size }\end{array}$ & Diagnosis & Design & Intervention \\
\hline Gibson, 2019 [128] & UK & $\begin{array}{l}\text { Nursing } \\
\text { students }\end{array}$ & $N=55$ & Self-harm & $\begin{array}{l}\text { Quasi-experimental } \\
\text { without control group }\end{array}$ & Lectures \\
\hline Happell, 2008 [129] & Australia & $\begin{array}{l}\text { Nursing } \\
\text { students }\end{array}$ & $N=687$ & Mixed & $\begin{array}{l}\text { Quasi-experimental } \\
\text { without control group }\end{array}$ & Expeditionary interventions \\
\hline Happell, 2019 [130] & $\begin{array}{l}\text { Australia, Ireland, } \\
\text { Finland }\end{array}$ & $\begin{array}{l}\text { Nursing } \\
\text { students }\end{array}$ & $N=194$ & Mixed & $\begin{array}{l}\text { Quasi-experimental } \\
\text { without control group }\end{array}$ & Contact-based interventions \\
\hline Hastings, 2017 [131] & USA & $\begin{array}{l}\text { Nursing } \\
\text { students }\end{array}$ & $N=310$ & Mixed & $\begin{array}{l}\text { Quasi-experimental } \\
\text { without control group }\end{array}$ & Expeditionary interventions \\
\hline Itzhaki, 2017 [132] & Israel & $\begin{array}{l}\text { Nursing } \\
\text { students }\end{array}$ & $N=101$ & Mixed & $\begin{array}{l}\text { Quasi-experimental } \\
\text { without control group }\end{array}$ & Contact-based interventions \\
\hline Kassam, 2011 [133] & UK & $\begin{array}{l}\text { Medical } \\
\text { students }\end{array}$ & $N=110$ & Mixed & $\begin{array}{l}\text { Quasi-experimental } \\
\text { with control group }\end{array}$ & Contact-based interventions \\
\hline Knaak, 2015 [134] & Canada & $\begin{array}{l}\text { Health } \\
\text { professionals }\end{array}$ & $N=191$ & $\begin{array}{l}\text { Borderline } \\
\text { personality } \\
\text { disorder }\end{array}$ & $\begin{array}{l}\text { Quasi-experimental } \\
\text { without control group }\end{array}$ & $\begin{array}{l}\text { Interventions targeting health } \\
\text { professionals }\end{array}$ \\
\hline Lam, 2011 [135] & Hong Kong & $\begin{array}{l}\text { General } \\
\text { practitioner }\end{array}$ & $N=69$ & Mixed & $\begin{array}{l}\text { Quasi-experimental } \\
\text { without control group }\end{array}$ & $\begin{array}{l}\text { Interventions targeting general } \\
\text { practitioners and medical doctors }\end{array}$ \\
\hline Lam, 2015 [136] & Hong Kong & $\begin{array}{l}\text { General } \\
\text { practitioners }\end{array}$ & $N=566$ & Mixed & $\begin{array}{l}\text { Quasi-experimental } \\
\text { with control group }\end{array}$ & $\begin{array}{l}\text { Interventions targeting general } \\
\text { practitioners and medical doctors }\end{array}$ \\
\hline Liekens, 2013 [137] & Belgium & Pharmacists & $N=141$ & Depression & RCT & Interventions targeting pharmacists \\
\hline Linville, 2013 [138] & USA & $\begin{array}{l}\text { General } \\
\text { practitioners }\end{array}$ & $N=45$ & Eating disorder & $\begin{array}{l}\text { Quasi-experimental } \\
\text { without control group }\end{array}$ & $\begin{array}{l}\text { Interventions targeting general } \\
\text { practitioners and medical doctors }\end{array}$ \\
\hline Lyons, 2015 [139] & Australia & $\begin{array}{l}\text { Medical } \\
\text { students }\end{array}$ & $N=151$ & Mixed & $\begin{array}{l}\text { Quasi-experimental } \\
\text { without control group }\end{array}$ & Expeditionary interventions \\
\hline $\begin{array}{l}\text { Manzanera, } 2018 \\
\text { [140] }\end{array}$ & Spain & $\begin{array}{l}\text { General } \\
\text { practitioners }\end{array}$ & $\begin{array}{l}N= \\
1322\end{array}$ & Depression & $\begin{array}{l}\text { Quasi-experimental } \\
\text { without control group }\end{array}$ & $\begin{array}{l}\text { Interventions targeting general } \\
\text { practitioners and medical doctors }\end{array}$ \\
\hline $\begin{array}{l}\text { Markstrom, } 2009 \\
{[141]}\end{array}$ & Sweden & $\begin{array}{l}\text { Health care } \\
\text { students }\end{array}$ & $N=167$ & Mixed & $\begin{array}{l}\text { Quasi-experimental } \\
\text { without control group }\end{array}$ & Expeditionary interventions \\
\hline $\begin{array}{l}\text { Martinez-Martinez, } \\
2019 \text { [142] }\end{array}$ & Spain & $\begin{array}{l}\text { Nursing } \\
\text { students }\end{array}$ & $N=185$ & Mixed & $\begin{array}{l}\text { Quasi-experimental } \\
\text { without control group }\end{array}$ & Contact-based interventions \\
\hline $\begin{array}{l}\text { McAllister, 2009a } \\
\text { [143] }\end{array}$ & Australia & $\begin{array}{l}\text { Emergency } \\
\text { personnel }\end{array}$ & $N=28$ & Self-harm & $\begin{array}{l}\text { Quasi-experimental } \\
\text { without control group }\end{array}$ & $\begin{array}{l}\text { Interventions targeting emergency } \\
\text { personnel }\end{array}$ \\
\hline $\begin{array}{l}\text { McAllister, 2009b } \\
\text { [144] }\end{array}$ & Australia & $\begin{array}{l}\text { Emergency } \\
\text { personnel }\end{array}$ & $N=36$ & Self-harm & $\begin{array}{l}\text { Quasi-experimental } \\
\text { without control group }\end{array}$ & $\begin{array}{l}\text { Interventions targeting emergency } \\
\text { personnel }\end{array}$ \\
\hline $\begin{array}{l}\text { Michalak, } 2014 \\
\text { [145] }\end{array}$ & Canada & $\begin{array}{l}\text { Health } \\
\text { professionals }\end{array}$ & $N=164$ & Bipolar disorder & $\begin{array}{l}\text { Quasi-experimental } \\
\text { without control group }\end{array}$ & $\begin{array}{l}\text { Interventions targeting health } \\
\text { professionals }\end{array}$ \\
\hline $\begin{array}{l}\text { Morrison, } 2009 \\
{[146]}\end{array}$ & Australia & $\begin{array}{l}\text { Nursing } \\
\text { students }\end{array}$ & N/A & Mixed & Qualitative & Contact-based interventions \\
\hline $\begin{array}{l}\text { Moxham, } 2016 \\
{[147]}\end{array}$ & Australia & $\begin{array}{l}\text { Nursing } \\
\text { students }\end{array}$ & $N=9$ & Mixed & $\begin{array}{l}\text { Quasi-experimental } \\
\text { with control group }\end{array}$ & Expeditionary interventions \\
\hline Muzyk, 2017 [148] & USA & $\begin{array}{l}\text { Pharmacy } \\
\text { students }\end{array}$ & $N=74$ & Mixed & $\begin{array}{l}\text { Quasi-experimental } \\
\text { without control group }\end{array}$ & Lectures \\
\hline $\begin{array}{l}\text { O' Connor, } 2013 \\
{[149]}\end{array}$ & Ireland & $\begin{array}{l}\text { Medical } \\
\text { students }\end{array}$ & $N=285$ & Mixed & $\begin{array}{l}\text { Quasi-experimental } \\
\text { without control group }\end{array}$ & Expeditionary interventions \\
\hline Omori, 2012 [150] & Japan & $\begin{array}{l}\text { Medical } \\
\text { doctors }\end{array}$ & $N=51$ & Schizophrenia & $\begin{array}{l}\text { Quasi-experimental } \\
\text { without control group }\end{array}$ & $\begin{array}{l}\text { Interventions targeting general } \\
\text { practitioners and medical doctors }\end{array}$ \\
\hline O'Reilly, 2010 [151] & Australia & $\begin{array}{l}\text { Pharmacy } \\
\text { students }\end{array}$ & $N=178$ & Mixed & $\begin{array}{l}\text { Quasi-experimental } \\
\text { without control group }\end{array}$ & Contact-based interventions \\
\hline O'Reilly, 2011 [152] & Australia & $\begin{array}{l}\text { Pharmacy } \\
\text { students }\end{array}$ & $N=60$ & Mixed & $\begin{array}{l}\text { Quasi-experimental } \\
\text { with control group }\end{array}$ & Lectures \\
\hline Papish, 2013 [153] & Canada & $\begin{array}{l}\text { Medical } \\
\text { students }\end{array}$ & $N=111$ & Mixed & RCT & Expeditionary interventions \\
\hline Patten, 2012 [154] & Canada & Pharmacy & $N=131$ & Mixed & RCT & Contact-based interventions \\
\hline
\end{tabular}


Table 4 Intervention studies (Continued)

\begin{tabular}{|c|c|c|c|c|c|c|}
\hline First author, year & Country & Population & $\begin{array}{l}\text { Sample } \\
\text { size }\end{array}$ & Diagnosis & Design & Intervention \\
\hline & & students & & & & \\
\hline Poreddi, 2015 [155] & India & $\begin{array}{l}\text { Medical } \\
\text { students }\end{array}$ & $N=176$ & Mixed & $\begin{array}{l}\text { Quasi-experimental } \\
\text { with control group }\end{array}$ & Lectures \\
\hline Romem, 2008 [156] & Israel & $\begin{array}{l}\text { Nursing } \\
\text { students }\end{array}$ & $N=126$ & Mixed & $\begin{array}{l}\text { Quasi-experimental } \\
\text { without control group }\end{array}$ & Expeditionary interventions \\
\hline Shen, 2014 [157] & China & $\begin{array}{l}\text { Medical } \\
\text { students }\end{array}$ & $N=325$ & Mixed & $\begin{array}{l}\text { Quasi-experimental } \\
\text { without control group }\end{array}$ & Expeditionary interventions \\
\hline Stacey, 2018 [158] & UK & $\begin{array}{l}\text { Nursing } \\
\text { students }\end{array}$ & N/A & Mixed & Qualitative & Lectures \\
\hline $\begin{array}{l}\text { Stuhlmiller, } 2019 \\
\text { [159] }\end{array}$ & USA & $\begin{array}{l}\text { Nursing } \\
\text { students }\end{array}$ & $N=85$ & Mixed & $\begin{array}{l}\text { Quasi-experimental } \\
\text { without control group }\end{array}$ & Expeditionary interventions \\
\hline $\begin{array}{l}\text { Telles-Correia, } 2015 \\
\text { [160] }\end{array}$ & Portugal & $\begin{array}{l}\text { Medical } \\
\text { students }\end{array}$ & $N=398$ & Mixed & $\begin{array}{l}\text { Quasi-experimental } \\
\text { without control group }\end{array}$ & Observational studies \\
\hline Treloar, 2009 [161] & Australia & $\begin{array}{l}\text { Emergency } \\
\text { personnel }\end{array}$ & $N=65$ & $\begin{array}{l}\text { Borderline } \\
\text { personality } \\
\text { disorder }\end{array}$ & $\begin{array}{l}\text { Quasi-experimental } \\
\text { with control group }\end{array}$ & $\begin{array}{l}\text { Interventions targeting emergency } \\
\text { personnel }\end{array}$ \\
\hline Upshur, 2008 [162] & USA & $\begin{array}{l}\text { General } \\
\text { practitioners }\end{array}$ & $N=9$ & Depression & $\begin{array}{l}\text { Quasi-experimental } \\
\text { without control group }\end{array}$ & $\begin{array}{l}\text { Interventions targeting general } \\
\text { practitioners and medical doctors }\end{array}$ \\
\hline Wang, 2016 [163] & Taiwan & $\begin{array}{l}\text { Medical } \\
\text { students }\end{array}$ & $N=72$ & Mixed & $\begin{array}{l}\text { Quasi-experimental } \\
\text { with control group }\end{array}$ & Observational studies \\
\hline Winkler, 2017 [164] & The Czech Republic & $\begin{array}{l}\text { Nursing } \\
\text { students }\end{array}$ & $N=499$ & Mixed & RCT & Contact-based interventions \\
\hline
\end{tabular}

Note: RCT = Randomized controlled trial, Observational studies = Observational studies of the effect of attending medical school

\section{Observational studies of the effect of attending medical school}

We identified five studies investigating the effect of attending medical school on stigmatizing attitudes and behaviors toward patients with mental disorders $[109,121,122,160,163]$. These studies were observational or based on natural experiments in contrast to the other studies. For instance, in Turkey researchers followed freshman medical students from 2008 to 2013. A questionnaire was administered to the participants on their first study year, before receiving any theoretical or practical training on psychiatry. Participants who completed their psychiatry internship were reassessed with a questionnaire five years later [121].

\section{Interventions targeting health professionals}

We identified 17 intervention studies targeting health professionals, including general practitioners and other medical doctors $(n=9)$, emergency personnel $(n=3)$, nurses $(n=1)$, pharmacists $(n=2)$, and nonspecific groups of health professionals $(n=2)$. We categorized these interventions into four intervention types: (1) Interventions targeting general practitioners and medical doctors, (2) Interventions targeting pharmacists, (3) Interventions targeting emergency personnel, and (4) Interventions targeting non-specific groups of health professionals.

\section{Interventions targeting general practitioners and medical} doctors

Nine studies focused on interventions targeting general practitioners and medical doctors [104, 111, 124, 135, 136, 138, 140, 150, 162]. They focused on attitudes and behaviors toward patients with specific mental disorders such as depression [111, 140, 162] or eating disorders [138]. The interventions differed considerably in content and scope. For example, in Hong Kong general practitioners participated in a 1-year part-time course. The course included 20 interactive seminars on mental disorders and 20 sessions visiting general practitioner consultations, including a written assignment. The seminars were developed and conducted by a family physician and a psychiatrist. After completing the seminars, the participants began clinical attachment in groups [135].

\section{Interventions targeting pharmacists}

We found two interventions targeting pharmacists $(n=$ 2 ), both of which addressed attitudes and behaviors toward patients with depression [113, 137]. The interventions aimed to empower pharmacists when encountering patients with depression through courses in communication skills, awareness of depression, and use of antidepressants. For example, Australian pharmacists were taught, by a psychiatrist, a psychologist, and a general practitioner, to give advice and support when dispensing 
medication. To upgrade their knowledge, the pharmacists received pamphlets on depression [113].

\section{Interventions targeting emergency personnel}

Few studies $(n=3)$ investigated the impact of educational programs on emergency personnel [143, 144, 161]. These interventions primarily focused on the reception of patients with mental disorders at emergency rooms through courses in evidence-based treatment and communication. For example, in Australia, researchers tested a 2-h lecture focusing on participants' attitudes and current practice in relation to self-harm. Lectures included theories for understanding self-harm and evidence-based treatment. Teaching material consisted of PowerPoint presentations and short video narratives from clinical practice and consumer reports [143].

\section{Interventions targeting non-specific groups of health professionals}

Three interventions did not target a specific group of health professionals, but included different professions $[108,134,145]$. These interventions differed considerably in content given that one was a lecture [108], one a workshop [134], and one a stage play [145]. In the latter, researchers from Canada worked closely with an actress and playwright who had bipolar disorder. They developed a one-woman stage play specifically targeting stigma toward that specific disorder. A director was hired for the rehearsal period. The audiences comprised people with bipolar disorder and health care providers working with this target group [145].

\section{Discussion}

The aim of this scoping review was to provide a comprehensive overview of the vast amount of literature within the research field of stigma toward people with a mental disorder in the somatic health care system. In total, we identified 137 studies, which include 73 observational studies and 64 intervention studies. We included qualitative and quantitative studies and a wide range of health care professionals and students. In the analysis, we described the characteristics of the studies and categorized them according to health profession and diagnosis. This contrasts with previous literature reviews that have typically focused more narrowly on, for instance, specific diagnoses or health care professionals ([23, 24, 165167], e.g.), and only a few previous reviews have had health care students as a target population [168, 169].

This scoping review created a comprehensive overview of the existing literature, employing a broad focus on both health care students and health professionals as well as inclusion of several mental disorders. This broad approach is helpful for identifying important knowledge gaps. All observational studies examined stigmatizing behaviors, attitudes, and/or perceptions among health professionals in the somatic health care system toward patients with mental disorders. We found that most observational studies $(n=13)$ focused on emergency personnel, followed by medical doctors $(n=10)$ and health professionals $(n=8)$, and that most studies $(n=$ 40) focused on several diagnoses. In studies focusing on single, specific diagnoses, the most frequent mental disorders were self-harm $(n=10)$ and schizophrenia $(n=9)$. Finally, we found that most of the observational studies used quantitative methods $(n=58)$, while 13 studies used qualitive methods and only two studies used mixed methods. Considering the complexity of the phenomena, more qualitative- and mixed method studies could deepen our understanding of stigma in somatic health care further.

With this review, we have provided insight into the distribution of studies in relation to the specific health profession and diagnosis that have dominated the literature, and which have been overlooked. We found that only a small number of all the included studies explicitly address stigma toward people with anxiety, bipolar disorder, and borderline personality disorder. This points to a need for future research to explore further the extent and characteristics of somatic health care professionals' stigmatizing attitudes and behaviors toward patients diagnosed with these disorders.

In general, the content of the included studies spans many different combinations of health professionals and diagnoses, confer Tables 1 and 2. Because of this diversity, it is not possible to conclude whether stigmatizing attitudes and behaviors may be more prevalent among some health care professions compared to others, and whether patients with a specific mental disorder are more exposed to stigmatization than others. However, this could be an interesting theme to investigate further in future research, as this knowledge can develop and strengthen anti-stigma campaigns targeting specific professions within the health sector.

We found that interventions to prevent or reduce stigma toward patients with mental disorders focused either on health care students or health care professionals. We categorized student interventions according to their content and the interventions targeting health professional according to the target group. In total, we identified four types of intervention studies targeting students (including lectures, expeditionary interventions, contact-based interventions, and observational studies of the effect of attending medical school) and four targeting health professionals (including general practitioners/medical doctors, pharmacists, emergency personnel and non-specific health professionals). The interventions varied in content and design, both within and across different target groups. Student interventions generally did not examine a specific mental 
disorder but rather multiple mental disorders or mental disorders in general, as opposed to interventions for health professionals, which often focused on both a specific target group and a specific diagnosis. This extended focus on intervention studies is, to our knowledge, not seen in previous reviews. Several previous reviews, however, call for more educational interventions to reduce negative attitudes and stigma among health professionals [167, 170], since more education and competency development can be associated with more positive attitudes [23, 171].

Following the scoping review methodology, we did not assess the quality of the studies included in the review, given that we focused on the overview of the literature. Thus, we cannot draw inferences about the effectiveness of interventions toward somatic health care professionals to reduce or prevent stigma. However, we did find that the quality of the effectiveness evaluations varied and that the majority used a quasi-experimental design (with or without a control group), while only eight studies employed an RCT. Therefore, this review points to a need for intervention studies with stronger evaluation designs.

Overall, our scoping review underlines the presence of stigmatizing attitudes and behaviors toward people with a mental disorder in the somatic health care system. The included studies show that stigma may be caused by several factors, e.g. lack of knowledge about mental disorders among health professionals, lack of time to care for more demanding or difficult patients and by health professionals' experiences of feeling insecure and unsafe in the presence of patients with mental disorders [111, 132, 152]. Following Link and Phelan's conceptualization of stigma, health professionals' experiences of lacking knowledge and competencies regarding mental disorders may initiate a stigmatization process in which they-due to dominant cultural beliefs-link undesirable characteristics and negative stereotypes to patients with mental disorders and engage in a separation of "us" from "them", leading the patients to experience status loss, discrimination and unequal outcomes and opportunities [12]. However, the identified intervention studies reveal that attitudes toward people with mental disorders among somatic health care professionals to a great extent reflect the attitudes of the general population [16, 108, 133]. This way, somatic health care professionals seem to exercise neither more nor less stigmatizing behavior than people in the general population. Importantly, and in contrast to the general population, health professionals possess power to determine the course, type and circumstances of treatment offered to patients with mental disorders. According to Link and Phelan (2001), power is precisely key to understanding stigma, because stigmatization is entirely contingent on access to social, economic and/or political power that allows the different elements of stigma to unfold [12]. Therefore, stigmatizing attitudes and behaviors toward mental disorders among health professionals constitute a critical problem that needs to be addressed both during their education and continuously throughout their professional career as part of their continuing professional development.

\section{Strengths and weaknesses}

This review has several limitations. First, as the search was limited to studies published after 2008, as well as studies in English or Nordic languages, we may have overlooked relevant studies and anti-stigmatizing interventions. Second, as mentioned above and as applies to scoping reviews in general, we did not assess the quality of the included studies.

Despite these limitations, we note several strengths of this study. First, a methodological strength is the fundamental systematic approach to charting the studies. We have systematically selected keywords and searched in selected databases based on several inclusion and exclusion criteria. Additionally, the screening process was undertaken on different levels: by title screening, abstract screening, and full-text screening in the review manager Covidence, after which all included studies were systematically organized and analyzed in NVivo. The use of NVivo contributed to a systematic organization of the included studies, which provided a useful tool for creating overview and high-level systematization of all included studies. Additionally, we consider it a strength that we have included intervention studies from the entire world. Although organization and practice within the somatic health care system are diverse worldwide, which reduces the transmissibility of the interventions, this knowledge contributes to the research field and can further inspire and mobilize new interventions. Finally, we consider it a strength that we have included a consultation element (e.g. stage 6) in our review process, as recommended by Arksey and O’Malley [28].

\section{Conclusion}

This scoping review has contributed to our knowledge about stigmatization of people with mental disorders in the somatic health care system. The large number of studies identified in this review suggests that stigmatizing attitudes and behaviors toward patients with mental disorders, within a somatic health care setting, is a worldwide challenge. By including studies focusing on different health professions and mental disorders, instead of focusing solely on a single profession or diagnosis as seen in many reviews, this review contributes with a more comprehensive overview.

The findings point to a need for further research on stigma toward patients with anxiety, bipolar disorder 
and borderline personality disorder. Furthermore, it would be beneficial to further knowledge on whether stigmatizing attitudes or behaviors toward mental disorders are more prevalent in some health care professions than others. Such knowledge could contribute to more targeted interventions.

\section{Supplementary Information}

The online version contains supplementary material available at https://doi. org/10.1186/s12888-021-03415-8.

\section{Additional file 1.}

\section{Acknowledgements}

Not applicable.

\section{Authors' contributions}

INS designed the research protocol and screened all articles. INS and AOK worked together to review the articles until agreement was reached. AOK and INS designed the tables. INS and AOK led the writing with contributions from MBDN. MBDN and PVP checked and revised the text. INS led the final editing stages of the manuscript. All authors read and approved the final manuscript.

\section{Funding}

We received funding from the organization EN AF OS. The organization took part in developing the research question and wasadditionally involved in qualifying keywords and search strings. The funding body had no role in the analysis, and interpretationof data and in writing the manuscript. The views expressed in this article are those of the authors and do not necessarily represent or reflect the views of the organization EN AF OS.

\section{Availability of data and materials}

All data generated or analyzed during this study are included in this published article. The search strategy is available as an additional file.

\section{Declarations}

\section{Ethical approval and consent to participate}

Not applicable.

\section{Consent for publication}

Not applicable.

\section{Competing interests}

The authors declare that they have no competing interests.

Received: 30 June 2021 Accepted: 6 August 2021

Published online: 07 September 2021

\section{References}

1. Whiteford HA, Degenhardt L, Rehm J, Baxter AJ, Ferrari AJ, Erskine HE, et al. Global burden of disease attributable to mental and substance use disorders: findings from the global burden of disease study 2010. Lancet. 2013;382(9904):1575-86.

2. Wittchen HU, Jacobi F, Rehm J, Gustavsson A, Svensson M, Jönsson B, et al. The size and burden of mental disorders and other disorders of the brain in Europe 2010. Eur Neuropsychopharmacol. 2011;21(9):655-79. https://doi. org/10.1016/j.euroneuro.2011.07.018.

3. Nordentoft M, Wahlbeck K, Hällgren J, Westman J, Ösby U, Alinaghizadeh H, et al. Excess mortality, causes of death and life expectancy in 270,770 patients with recent onset of mental disorders in Denmark, Finland and Sweden. PLoS One. 2013;8(1):e55176. https://doi.org/10.1371/journal.pone. 0055176.

4. Walker ER, McGee RE, Druss BG. Mortality in mental disorders and global disease burden implications: a systematic review and Meta-analysis. JAMA Psychiatry. 2015;72(4):334-41. https://doi.org/10.1001/jamapsychiatry.2014.2 502.
5. Kugathasan P, Stubbs B, Aagaard J, Jensen SE, Munk Laursen T, Nielsen RE. Increased mortality from somatic multimorbidity in patients with schizophrenia: a Danish nationwide cohort study. Acta Psychiatr Scand. 2019;140(4):340-8.

6. Laursen TM, Munk-Olsen T, Gasse C. Chronic somatic comorbidity and excess mortality due to natural causes in persons with schizophrenia or bipolar affective disorder. PLoS One. 2011;6(9):e24597. https://doi.org/10.13 71/journal.pone.0024597.

7. Stubbs B, Vancampfort D, Veronese N, Kahl KG, Mitchell AJ, Lin PY, et al. Depression and physical health multimorbidity: primary data and country-wide meta-analysis of population data from 190593 people across 43 low- and middle-income countries. Psychol Med. 2017:47(12):2107-17.

8. Stone EM, Chen LN, Daumit GL, Linden S, McGinty EE. General medical Clinicians' attitudes toward people with serious mental illness: a scoping review. J Behav Health Serv Res. 2019;46(4):656-79. https://doi.org/10.1007/ s11414-019-09652-w.

9. Mitchell AJ, Santo Pereira IE, Yadegarfar M, Pepereke S, Mugadza V, Stubbs B. Breast cancer screening in women with mental illness: comparative metaanalysis of mammography uptake. Br J Psychiatry. 2014;205(6):428-35. https://doi.org/10.1192/bjp.bp.114.147629.

10. Lord O, Malone D, Mitchell AJ. Receipt of preventive medical care and medical screening for patients with mental illness: a comparative analysis. Gen Hosp Psychiatry. 2010;32(5):519-43.

11. Havercamp SM, Scott HM. National health surveillance of adults with disabilities, adults with intellectual and developmental disabilities, and adults with no disabilities. Disabil Health J. 2015;8(2):165-72. https://doi. org/10.1016/j.dhjo.2014.11.002.

12. Link BG, Phelan JC. Conceptualizing stigma. Annu Rev Sociol. 2001;27(1): 363-85.

13. Nash M. Diagnostic overshadowing: a potential barrier to physical health care for mental health service users. Ment Health Pract. 2013;17(4):22-6. https://doi.org/10.7748/mhp2013.12.17.4.22.e862.

14. Thornicroft G. Physical health disparities and mental illness: the scandal of premature mortality. Br J Psychiatry. 2011;199(6):441-2.

15. Shefer G, Henderson C, Howard LM, Murray J, Thornicroft G. Diagnostic overshadowing and other challenges involved in the diagnostic process of patients with mental illness who present in emergency departments with physical symptoms--a qualitative study. PLoS One. 2014;9(11):e111682. https://doi.org/10.1371/journal.pone.0111682.

16. Knaak S, Mantler E, Szeto A. Mental illness-related stigma in healthcare: barriers to access and care and evidence-based solutions. Healthcare Manage Forum. 2017;30(2):111-6. https://doi.org/10.1177/0840470416679413.

17. Clement S, Schauman O, Graham T, Maggioni F, Evans-Lacko S, Bezborodovs $\mathrm{N}$, et al. What is the impact of mental health-related stigma on help-seeking? A systematic review of quantitative and qualitative studies. Psychol Med. 2015;45(1):11-27.

18. Mitchell AJ, Malone D, Doebbeling CC. Quality of medical care for people with and without comorbid mental illness and substance misuse: systematic review of comparative studies. Br J Psychiatry. 2009;194(6):491-9. https://doi. org/10.1192/bjp.bp.107.045732.

19. Kilbourne AM, Welsh D, McCarthy JF, Post EP, Blow FC. Quality of care for cardiovascular disease-related conditions in patients with and without mental disorders. J Gen Intern Med. 2008;23(10):1628-33. https://doi.org/1 0.1007/s11606-008-0720-z.

20. Lawrence D, Kisely S. Inequalities in healthcare provision for people with severe mental illness. J Psychopharmacol (Oxford, England). 2010;24(4 Suppl):61-8

21. Rayner G, Blackburn J, Edward KL, Stephenson J, Ousey K. Emergency department nurse's attitudes towards patients who self-harm: a metaanalysis. Int J Ment Health Nurs. 2019;28(1):40-53.

22. Giandinoto JA, Stephenson J, Edward KL. General hospital health professionals' attitudes and perceived dangerousness towards patients with comorbid mental and physical health conditions: systematic review and meta-analysis. Int J Ment Health Nurs. 2018;27(3):942-55.

23. Karman P, Kool N, Poslawsky IE, van Meijel B. Nurses' attitudes towards selfharm: a literature review. J Psychiatr Ment Health Nurs. 2015;22(1):65-75. https://doi.org/10.1111/jpm.12171.

24. Alexander V, Ellis H, Barrett B. Medical-surgical Nurses' perceptions of psychiatric patients: a review of the literature with clinical and practice applications. Arch Psychiatr Nurs. 2016;30(2):262-70. 
25. Munn Z, Peters MDJ, Stern C, Tufanaru C, McArthur A, Aromataris E. Systematic review or scoping review? Guidance for authors when choosing between a systematic or scoping review approach. BMC Med Res Methodol. 2018;18(1):143.

26. Peters MDJ, Godfrey CM, Khalil H, McInerney P, Parker D, Soares CB. Guidance for conducting systematic scoping reviews. Int J Evid Based Healthcare. 2015;13(3):141-6.

27. Tricco AC, Lillie E, Zarin W, O'Brien KK, Colquhoun H, Levac D, et al. PRISMA extension for scoping reviews (PRISMA-SCR): checklist and explanation. Ann Intern Med. 2018;169(7):467-73. https://doi.org/10.7326/M18-0850.

28. Arksey H, O'Malley L. Scoping studies: towards a methodological framework. Int J Soc Res Methodol. 2005;8(1):19-32.

29. Concannon TW, Grant S, Welch V, Petkovic J, Selby J, Crowe S, et al. Practical guidance for involving stakeholders in Health Research. J Gen Intern Med. 2019;34(3):458-63. https://doi.org/10.1007/s11606-018-4738-6.

30. Abood A, Jain S, Zhong J. Self-harm and the surgeon. J Plastic Reconstruct Aesthetic Surg. 2009;62(5):e106-7.

31. Anderson K, Accurso E, Kinasz K, Grange D, Accurso EC, Kinasz KR, et al. Residents' and Fellows' knowledge and attitudes about eating disorders at an Academic Medical Center. Acad Psychiatry. 2017:41(3):381-4.

32. Arbanas $G$, Rožman J, Bagarić Š. The attitudes of medical doctors, nurses and lay people towards schizophrenia, depression and PTSD. Psychiatr Danub. 2019;31(Suppl 1):84-91.

33. Artis L, Smith JR. Emergency department staff attitudes toward people who self-harm: exploring the influences of norms and identity. Adv Emerg Nurs J. 2013;35(3):259-69.

34. Arvaniti A, Samakouri M, Kalamara E, Bochtsou V, Bikos C, Livaditis M. Health service staff's attitudes towards patients with mental illness. Soc Psychiatry Psychiatr Epidemiol. 2009;44(8):658-65.

35. Avery JD, Taylor KE, Kast KA, Kattan J, Gordon-Elliot J, Mauer E, et al. Attitudes Toward Individuals With Mental IIIness and Substance Use Disorders Among Resident Physicians. Primary Care Companion CNS Disord. 2019;21(1).

36. Bannatyne AJ, Stapleton PB. Attitudes towards anorexia nervosa: volitional stigma differences in a sample of pre-clinical medicine and psychology students. J Mental Health (Abingdon, England). 2017;26(5):442-8.

37. Bell JS, Aaltonen SE, Airaksinen MS, Volmer D, Gharat MS, Muceniece R, et al. Determinants of mental health stigma among pharmacy students in Australia, Belgium, Estonia, Finland, India and Latvia. Int J Soc Psychiatry. 2010;56(1):3-14.

38. Bell JS, Aaltonen SE, Bronstein E, Desplenter FA, Foulon V, Vitola A, et al. Attitudes of pharmacy students toward people with mental disorders, a six country study. Pharm World Sci. 2008;30(5):595-9.

39. Bjorkman T, Angelman T, Jonsson M. Attitudes towards people with mental illness: a cross-sectional study among nursing staff in psychiatric and somatic care. Scand J Caring Sci. 2008;22(2):170-7.

40. Brunero S, Buus N, West S. Categorising patients mental illness by medical surgical nurses in the general hospital Ward: a focus group study. Arch Psychiatr Nurs. 2017;31(6):614-23.

41. Castillejos Anguiano MC, Bordallo Aragon A, Aguilera Fernandez D, Moreno Kustner B. Perceptions about mental illness among general practitioners. Int J Ment Heal Syst. 2019;13:27.

42. Ceylan B, Kocoglu-Tanyer D. An examination of Turkish Nurses' attitudes, awareness and practices regarding reproductive health needs of individuals with schizophrenia. Mental Health Nursing. 2019;40(5).

43. Chapman R, Martin C. Perceptions of Australian emergency staff towards patients presenting with deliberate self-poisoning: a qualitative perspective. Int Emerg Nurs. 2014;22(3):140-5.

44. Clifton A, Burgess C, Clement S, Ohlsen R, Ramluggun P, Sturt J, et al. Influences on uptake of cancer screening in mental health service users: a qualitative study. BMC Health Serv Res. 2016;16:257.

45. Conlon M, O'Tuathail C. Measuring emergency department nurses' attitudes towards deliberate self-harm using the self-harm antipathy scale. Int Emerg Nurs. 2012;20(1):3-13. https://doi.org/10.1016/j.ienj.2010.08.001.

46. Crapanzano K, Fisher D, Hammarlund R, Hsieh EP, May W. An exploration of Residents' implicit biases towards depression-a pilot study. J Gen Intern Med. 2018:33(12):2065-9.

47. Currin L, Waller G, Schmidt U. Primary care physicians' knowledge of and attitudes toward the eating disorders: do they affect clinical actions? Int J Eat Disord. 2009;42(5):453-8. https://doi.org/10.1002/eat.20636.

48. Cutler JL, Harding KJ, Mozian SA, Wright LL, Pica AG, Masters SR, et al. Discrediting the notion "working with 'crazies' will make you 'crazy'": addressing stigma and enhancing empathy in medical student education. Adv Health Sci Educ Theory Pract. 2009;14(4):487-502.
49. Dixon RP, Roberts LM, Lawrie S, Jones LA, Humphreys MS. Medical students' attitudes to psychiatric illness in primary care. Med Educ. 2008;42(11):1080-7.

50. Ewalds-Kvist B, Hogberg T, Lutzen K. Student nurses and the general population in Sweden: trends in attitudes towards mental illness. Nordic J Psychiatry. 2013;67(3):164-70.

51. Gawley L, Einarson A, Bowen A. Stigma and attitudes towards antenatal depression and antidepressant use during pregnancy in healthcare students. Adv Health Sci Educ Theory Pract. 2011;16(5):669-79. https://doi. org/10.1007/s10459-011-9289-0

52. Giannetti V, Caley CF, Kamal KM, Covvey JR, McKee J, Wells BG, et al. Community pharmacists and mental illness: a survey of service provision, stigma, attitudes and beliefs. Int J Clin Pharm. 2018;40(5):1096-105. https://doi.org/10.1007/s11096-018-0619-7.

53. Gordon JT. Emergency department junior medical staff's knowledge, skills and confidence with psychiatric patients: a survey. Psychiatrist. 2012;36(5):186-8.

54. Granados-Gamez G, Lopez Rodriguez MDM, Corral Granados A, MarquezHernandez W. Attitudes and beliefs of nursing students toward mental disorder: the significance of direct experience with patients. Perspect Psychiatric Care. 2017;53(2):135-43.

55. Happell B, Robins A, Gough K. Developing more positive attitudes towards mental health nursing in undergraduate students: part 2--the impact of theory and clinical experience. J Psychiatr Ment Health Nurs. 2008;15(7):527-36.

56. Happell B, Platania-Phung C, Bocking J, Scholz B, Horgan A, Manning F, et al. Nursing Students' attitudes towards people diagnosed with mental illness and mental health nursing: an international project from Europe and Australia. Issues Mental Health Nurs. 2018;39(10):829-39.

57. Heyward-Chaplin J, Shepherd L, Arya R, O'Boyle CP. Audit of healthcare professionals' attitudes towards patients who self-harm and adherence to national guidance in a UK burns and plastic surgery department. Scars Burn Heal. 2018:4:2059513118764100.

58. Ihalainen-Tamlander N, Vahaniemi A, Loyttyniemi E, Suominen T, Valimaki M. Stigmatizing attitudes in nurses towards people with mental illness: a cross-sectional study in primary settings in Finland. J Psychiatr Ment Health Nurs. 2016;23(6-7):427-37. https://doi.org/10.1111/jpm.12319.

59. Janouskova M, Weissova A, Formanek T, Pasz J, Bankovska Motlova L. Mental illness stigma among medical students and teachers. Int J Soc Psychiatry. 2017;63(8):744-51. https://doi.org/10.1177/0020764017735347.

60. Jones SM, Vahia IV, Cohen Cl, Hindi A, Nurhussein M. A pilot study to assess attitudes, behaviors, and inter-office communication by psychiatrists and primary care providers in the care of older adults with schizophrenia. Int J Geriatric Psychiatry. 2009;24(3):254-60.

61. Koning KL, McNaught A, Tuffin K. Emergency department staff beliefs about self-harm: a thematic framework analysis. Community Ment Health J. 2018; 54(6):814-22.

62. Thongpriwan V, Leuck SE, Powell RL, Young S, Schuler SG, Hughes RG. Undergraduate nursing students' attitudes toward mental health nursing. Nurse education today. 2015:35(8):948-53.

63. Kopera M, Suszek H, Bonar E, Myszka M, Gmaj B, Ilgen M, et al. Evaluating explicit and implicit stigma of mental illness in mental health professionals and medical students. Community Ment Health J. 2015;51(5):628-34.

64. Korszun A, Dinos S, Ahmed K, Bhui K. Medical student attitudes about mental illness: does medical-school education reduce stigma? Acad Psychiatry. 2012;36(3):197-204. https://doi.org/10.1176/appi.ap.10110159.

65. Kuzman MR, Bosnjak D, Vokal P, Kuharic J, Brkic I, Kuzman T, et al. Can medical students recognize depression? A survey at the Zagreb School of Medicine. Acad Psychiatry. 2014;38(3):312-5.

66. Leddy MA, Jones C, Morgan MA, Schulkin J. Eating disorders and obstetricgynecologic care. J Women's Health (Larchmt). 2009;18(9):1395-401.

67. Liekens S, Smits T, Laekeman G, Foulon V. Factors determining social distance toward people with depression among community pharmacists. Eur Psychiatry. 2012;27(7):528-35.

68. Magliano L, Read J, Rega S, Oliviero N, Sagliocchi A, Patalano M, et al. The influence of causal explanations and diagnostic labeling on medical students' views of schizophrenia. Acad Med. 2011;86(9):1155-62.

69. Magliano L, Strino A, Punzo R, Acone R, Affuso G, Read J. Effects of the diagnostic label 'schizophrenia', actively used or passively accepted, on general practitioners' views of this disorder. Int J Soc Psychiatry. 2017;63(3): 224-34

70. McCann TV, Savic M, Ferguson N, Cheetham A, Witt K, Emond K, et al. Recognition of, and attitudes towards, people with depression and psychosis with/without alcohol and other drug problems: results from a 
national survey of Australian paramedics. BMJ Open. 2018;8(12):e023860. https://doi.org/10.1136/bmjopen-2018-023860.

71. McCarthy L, Gijbels H. An examination of emergency department nurses' attitudes towards deliberate self-harm in an Irish teaching hospital. Int Emerg Nurs. 2010;18(1):29-35. https://doi.org/10.1016/j.ienj.2009.05.005.

72. Morral K, Morral J. A survey of community pharmacists' attitudes towards mental illness. J Public Ment Health. 2016;15(2):93-102.

73. Muehlenkamp JJ, Claes L, Quigley K, Prosser E, Claes S, Jans D. Association of training on attitudes towards self-injuring clients across health professionals. Arch Suicide Res. 2013;17(4):462-8.

74. Nauta K, Boenink AD, Wimalaratne IK, Menkes DB, Mellsop G, Broekman B. Attitudes of general hospital consultants towards psychosocial and psychiatric problems in Netherlands. Psychol Health Med. 2019;24(4):402-13. https://doi.org/10.1080/13548506.2018.1546020.

75. Neauport A, Rodgers RF, Simon NM, Birmes PJ, Schmitt L, Bui E. Effects of a psychiatric label on medical residents' attitudes. Int I Soc Psychiatry. 2012; 58(5):485-7. https://doi.org/10.1177/0020764011408652.

76. Noonan M, Jomeen J, Galvin R, Doody O. Survey of midwives' perinatal mental health knowledge, confidence, attitudes and learning needs. Women Birth. 2018;31(6):e358-66. https://doi.org/10.1016/j.wombi.2018.02.002.

77. Nutt R, Gilchrist G, Marsa-Sambola F, Baldacchino A. Staff regard towards working with patients with co-morbid depression and substance misuse: an exploratory study. Heroin Addiction Relat Clin Problems. 2017;19(2):5-16.

78. O'Reilly CL, Bell JS, Chen TF. Mental health consumers and caregivers as instructors for health professional students: a qualitative study. Soc Psychiatry Psychiatric Epidemiol. 2012;47(4):607-13.

79. O'Reilly CL, Bell JS, Kelly PJ, Chen TF. Exploring the relationship between mental health stigma, knowledge and provision of pharmacy services for consumers with schizophrenia. Res Soc Adm Pharm. 2015;11(3):e101-9.

80. Peitl MV, Peitl V, Pavlović E, Proloscić J, Petrić D. Stigmatization of patients suffering from schizophrenia. Coll Antropol. 2011;35(Suppl 2):141-5.

81. Perboell PW, Hammer NM, Oestergaard B, Konradsen H. Danish emergency nurses' attitudes towards self-harm - a cross-sectional study. Int Emerg Nurs. 2015;23(2):144-9.

82. Prener C, Lincoln AK. Emergency medical services and "psych calls": examining the work of urban EMS providers. Am J Orthopsychiatry. 2015; 85(6):612-9.

83. Rai L, Shepherd L, O'Boyle CP. Quantitative and thematic analysis of burns surgeons' attitudes, beliefs and surgical decision-making in self-harm burn injuries: the use of a questionnaire and hypothetical cases. Burns. 2019;45(1): 180-9. https://doi.org/10.1016/j.burns.2018.08.003.

84. Rao H, Mahadevappa H, Pillay P, Sessay M, Abraham A, Luty J. A study of stigmatized attitudes towards people with mental health problems among health professionals. J Psychiatr Ment Health Nurs. 2009;16(3):279-84.

85. Raveneau G, Feinstein R, Rosen LM, Fisher M. Attitudes and knowledge levels of nurses and residents caring for adolescents with an eating disorder. Int J Adolescent Med Health. 2014;26(1):131-6.

86. Reavley NJ, Mackinnon AJ, Morgan AJ, Jorm AF. Stigmatising attitudes towards people with mental disorders: a comparison of Australian health professionals with the general community. Aust New Zealand J Psychiatry. 2014;48(5):433-41.

87. Rickles NM, Dube GL, McCarter A, Olshan JS. Relationship between attitudes toward mental illness and provision of pharmacy services. J Am Pharmacists Assoc. 2010;50(6):704-13.

88. Sandhu HS, Arora A, Brasch J, Streiner DL. Mental health stigma: explicit and implicit attitudes of Canadian undergraduate students, medical school students, and psychiatrists. Can J Psychiatr. 2019;64(3):209-17.

89. Schafer T, Wood S, Williams R. A survey into student nurses' attitudes towards mental illness: implications for nurse training. Nurse Educ Today. 2011;31(4):328-32.

90. Schmidt HG, Van Gog T, Schuit SC, Van den Berge K, Van Daele PL, Bueving $H$, et al. Do patients' disruptive behaviours influence the accuracy of a doctor's diagnosis? A randomised experiment. BMJ Qual Saf. 2017;26(1):19-23.

91. Serafini G, Pompili M, Haghighat R, Pucci D, Pastina M, Lester D, et al. Stigmatization of schizophrenia as perceived by nurses, medical doctors, medical students and patients. J Psychiatr Ment Health Nurs. 2011;18(7): 576-85.

92. Stumbo SP, Yarborough BJH, Yarborough MT, Green CA. Perspectives on providing and receiving preventive health care from primary care providers and their patients with mental illnesses. Am J Health Promot. 2018;32(8): 1730-9.
93. Treloar AJ. A qualitative investigation of the clinician experience of working with borderline personality disorder. N Z J Psychol. 2009;38(2):30-4.

94. van Nieuwenhuizen A, Henderson C, Kassam A, Graham T, Murray J, Howard $L M$, et al. Emergency department staff views and experiences on diagnostic overshadowing related to people with mental illness. Epidemiol Psychiatric Sci. 2013;22(3):255-62. https://doi.org/10.1017/S2045796012000571.

95. Volmer D, Maesalu M, Bell JS. Pharmacy students' attitudes toward and professional interactions with people with mental disorders. Int I Soc Psychiatry. 2008;54(5):402-13. https://doi.org/10.1177/0020764008090427.

96. Weare R, Green C, Olasoji M, Plummer V. ICU nurses feel unprepared to care for patients with mental illness: a survey of nurses' attitudes, knowledge, and skills. Intensive Crit Care Nurs. 2019;53:37-42.

97. Winkler P, Mlada K, Janouskova M, Weissova A, Tuskova E, Csemy L, et al. Attitudes towards the people with mental illness: comparison between Czech medical doctors and general population. Soc Psychiatry Psychiatr Epidemiol. 2016;51(9):1265-73.

98. Woollaston K, Hixenbaugh P. Destructive whirlwind: nurses' perceptions of patients diagnosed with borderline personality disorder. J Psychiatr Ment Health Nurs. 2008;15(9):703-9. https://doi.org/10.1111/j.1365-2850.2008.01275.x.

99. Yildirim M, Demirbuken I, Balci B, Yurdalan U. Beliefs towards mental illness in Turkish physiotherapy students. Physiother Theory Pract. 2015;31(7):461-5.

100. Zolnierek CD, Clingerman EM. A medical-surgical nurse's perceptions of caring for a person with severe mental illness. J Am Psychiatric Nurses Assoc. 2012;18(4):226-35.

101. Airagnes G, Consoli SM, De Morlhon O, Galliot A-M, Lemogne C, Jaury P. Appropriate training based on Balint groups can improve the empathic abilities of medical students: a preliminary study. J Psychosom Res. 2014; 76(5):426-9. https://doi.org/10.1016/j.jpsychores.2014.03.005.

102. Arbanas G, Bosnjak D, Sabo T. Impact of a nursing in psychiatry course on Students' attitudes toward mental health disorders. J Psychosoc Nurs Ment Health Serv. 2018:56(3):45-51.

103. Bannatyne A, Stapleton P. Educating medical students about anorexia nervosa: a potential method for reducing the volitional stigma associated with the disorder. Eat Disord. 2015;23(2):115-33.

104. Beaulieu T, Lauria-Horner B, Patten S, Knaak S, Weinerman R, Campbell H. Impact of skill-based approaches in reducing stigma in primary care physicians: results from a double-blind, parallel-cluster, randomized controlled trial. Can J Psychiatr. 2017;62(5):327-35. https://doi.org/10.1177/ 0706743716686919.

105. Bilge A, Palabiyik O. The effect of short films about mental health and disorders on preventing stigmatization in nursing education. Arch Psychiatr Nurs. 2017;31(1):88-92.

106. Bingham H, O'Brien AJ. Educational intervention to decrease stigmatizing attitudes of undergraduate nurses towards people with mental illness. Int J Ment Health Nurs. 2018;27(1):311-9.

107. Brenner AM. What medical students say about psychiatry: results of a reflection exercise. Acad Psychiatry. 2011;35(3):196-8. https://doi.org/10.11 76/appi.ap.35.3.196.

108. Calloway SJ, Young CS, Ward-Smith P, Paulsen L. Management of mental disorders in primary care: the impact of case based learning on nurse practitioner student role perception and stigmatizing attitudes. Nurse Educ Pract. 2017;25:43-9. https://doi.org/10.1016/j.nepr.2017.04.009.

109. Chiles C, Stefanovics E, Rosenheck R. Attitudes of students at a US medical school toward mental illness and its causes. Acad Psychiatry. 2017:41(3):320-5

110. Clement S, van Nieuwenhuizen A, Kassam A, Flach C, Lazarus A, de Castro $\mathrm{M}$, et al. Filmed $\mathrm{v}$. live social contact interventions to reduce stigma: randomised controlled trial. Br J Psychiatry. 2012;201(1):57-64.

111. Coppens E, Van Audenhove C, Gusmao R, Purebl G, Szekely A, Maxwell M, et al. Effectiveness of general practitioner training to improve suicide awareness and knowledge and skills towards depression. J Affect Disord. 2018;227:17-23. https://doi.org/10.1016/j.jad.2017.09.039.

112. Crisafulli MA, Von Holle A, Bulik CM. Attitudes towards anorexia nervosa: the impact of framing on blame and stigma. Int J Eat Disord. 2008:41(4):333-9. https://doi.org/10.1002/eat.20507.

113. Crockett J, Taylor S. Rural pharmacist perceptions of a project assessing their role in the management of depression. Aust J Rural Health. 2009;17(5):236-43.

114. Demiroren M, Saka MC, Senol Y, Senturk V, Baysal O, Oztuna D. The impact of reflective practices on medical students' attitudes towards mental illness. Anadolu Psikiyatri Dergisi. 2016;17(6):466-75. 
115. Dipaula BA, Qian J, Mehdizadegan N, Simoni-Wastila L. An elective psychiatric course to reduce pharmacy students' social distance toward people with severe mental illness. Am J Pharm Educ. 2011;75(4):72.

116. Duffy ME, Henkel KE. Words on walls: passive eating disorder education. Eat Disord. 2016;24(2):148-60.

117. Duman Z, Günüşen NP, İnan F, Ince S, Sari A. Effects of two different psychiatric nursing courses on nursing students' attitudes towards mental illness, perceptions of psychiatric nursing, and career choices. J Prof Nurs. 2017;33(6):452-9. https://doi.org/10.1016/j.profnurs.2017.06.005.

118. Economou M, Kontoangelos K, Peppou LE, Arvaniti A, Samakouri M, Douzenis A, et al. Medical students' attitudes to mental illnesses and to psychiatry before and after the psychiatric clerkship: training in a specialty and a general hospital. Psychiatry Res. 2017;258:108-15.

119. Economou M, Peppou LE, Louki E, Stefanis CN. Medical students' beliefs and attitudes towards schizophrenia before and after undergraduate psychiatric training in Greece. Psychiatry Clin Neurosci. 2012;66(1):17-25.

120. Eksteen H-C, Becker PJ, Lippi G. Stigmatization towards the mentally ill: perceptions of psychiatrists, pre-clinical and post-clinical rotation medical students. Int J Soc Psychiatry. 2017;63(8):782-91. https://doi.org/10.1177/002 0764017735865.

121. Esen Danacı A, Balıkçı K, Aydın O, Cengisiz C, Uykur AB. The effect of medical education on attitudes towards schizophrenia: a five-year follow-up study. Turk Psikiyatri Derg. 2016;27(3):176-84.

122. Failde I, Salazar A, Elorza J, Casais L, Pérez V, Martínez LC, et al. Spanish medical students' attitudes and views towards mental health and psychiatry: a multicentric cross-sectional study. Acad Psychiatry. 2014;38(3): 332-8.

123. Fernandez A, Tan KA, Knaak S, Chew BH, Ghazali SS. Effects of brief psychoeducational program on stigma in Malaysian pre-clinical medical students: a randomized controlled trial. Acad Psychiatry. 2016;40(6):905-11. https://doi.org/10.1007/s40596-016-0592-1.

124. Flanagan EH, Buck T, Gamble A, Hunter C, Sewell I, Davidson L. "Recovery Speaks": A Photovoice Intervention to Reduce Stigma Among Primary Care Providers. Psychiatric Serv (Washington, DC). 2016:67(5):566-9.

125. Fokuo JK, Goldrick V, Rossetti J, Wahlstrom C, Kundert C, Larson J, et al. Decreasing the stigma of mental illness through a student-nurse mentoring program: a qualitative study. Community Ment Health J. 2017;53(3):257-65.

126. Gable KN, Muhlstadt KL, Celio MA. A mental health elective to improve pharmacy students' perspectives on mental illness. Am J Pharm Educ. 2011; 75(2):34. https://doi.org/10.5688/ajpe75234.

127. Galletly C, Burton C. Improving medical student attitudes towards people with schizophrenia. Aust New Zealand J Psychiatry. 2011;45(6):473-6.

128. Gibson R, Carson J, Houghton T. Stigma towards non-suicidal self-harm: evaluating a brief educational intervention. Br J Nurs (Mark Allen Publishing). 2019;28(5):307-12. https://doi.org/10.12968/bjon.2019.28.5.307.

129. Happell B. The importance of clinical experience for mental health nursing part 1: undergraduate nursing students' attitudes, preparedness and satisfaction. Int J Ment Health Nurs. 2008;17(5):326-32.

130. Happell B, Platania-Phung C, Scholz B, Bocking J, Horgan A, Manning F, et al. Changing attitudes: the impact of expert by experience involvement in mental health nursing education: an international survey study. Int J Ment Health Nurs. 2019;28(2):480-91.

131. Hastings T, Kroposki M, Williams G. Can completing a mental health nursing course change Students' attitudes? Issues Mental Health Nurs. 2017;38(5):449-54.

132. Itzhaki M, Meridan O, Sagiv-Schifter T, Barnoy S. Nursing Students' attitudes and intention to work with mentally ill patients before and after a planned intervention. Acad Psychiatry. 2017:41(3):337-44.

133. Kassam A, Glozier N, Leese M, Loughran J, Thornicroft G. A controlled trial of mental illness related stigma training for medical students. BMC Med Educ. 2011;11:51.

134. Knaak S, Szeto AC, Fitch K, Modgill G, Patten S. Stigma towards borderline personality disorder: effectiveness and generalizability of an anti-stigma program for healthcare providers using a pre-post randomized design. Borderline personality disorder and emotion dysregulation. 2015;2(1):9. https://doi.org/10.1186/s40479-015-0030-0.

135. Lam TP, Goldberg D, Tse EYY, Lam KF, Mak KY, Lam EWW. What do primary care doctors get out of a year-long postgraduate course in community psychological medicine? Int J Psychiatry Med. 2011;42(2):133-49. https://doi. org/10.2190/PM.42.2.C.

136. Lam TP, Lam KF, Lam EWW, Sun KS. Does postgraduate training in community mental health make a difference to primary care Physicians' attitudes to depression and schizophrenia? Community Ment Health J. 2015; 51(6):641-6. https://doi.org/10.1007/s10597-015-9829-9.

137. Liekens S, Smits T, Laekeman G, Foulon V. A depression training session with consumer educators to reduce stigmatizing views and improve pharmacists' depression care attitudes and practices. Am J Pharm Educ. 2013;77(6):120.

138. Linville D, Aoyama T, Knoble NB, Gau J. The effectiveness of a brief eating disorder training programme in medical settings. J Res Nurs. 2013;18(6):54458. https://doi.org/10.1177/1744987112452182.

139. Lyons Z, Janca A. Impact of a psychiatry clerkship on stigma, attitudes towards psychiatry, and psychiatry as a career choice. BMC Med Educ. 2015; 15:34.

140. Manzanera R, Lahera G, Alvarez-Mon MA, Alvarez-Mon M. Maintained effect of a training program on attitudes towards depression in family physicians. Fam Pract. 2018;35(1):61-6. https://doi.org/10.1093/fampra/cmx071.

141. Markstrom U, Gyllensten AL, Bejerholm U, Bjorkman T, Brunt D, Hansson L, et al. Attitudes towards mental illness among health care students at Swedish universities--a follow-up study after completed clinical placement. Nurse Educ Today. 2009;29(6):660-5.

142. Martinez-Martinez C, Sanchez-Martinez V, Sales-Orts R, Dinca A, RichartMartinez M, Ramos-Pichardo JD. Effectiveness of direct contact intervention with people with mental illness to reduce stigma in nursing students. Int J Ment Health Nurs. 2019;28(3):735-43. https://doi.org/10.1111/inm.12578.

143. McAllister M, Billett S, Moyle W, Zimmer-Gembeck M. Use of a think-aloud procedure to explore the relationship between clinical reasoning and solution-focused training in self-harm for emergency nurses. J Psychiatr Ment Health Nurs. 2009a;16(2):121-8. https://doi.org/10.1111/j.1365-2850.2 008.01339.x.

144. McAllister M, Moyle W, Billett S, Zimmer-Gembeck M. 'I can actually talk to them now': qualitative results of an educational intervention for emergency nurses caring for clients who self-injure. J Clin Nurs. 2009b;18(20):2838-45. https://doi.org/10.1111/j.1365-2702.2008.02540.x.

145. Michalak EE, Livingston JD, Maxwell V, Hole R, Hawke LD, Parikh SV. Using theatre to address mental illness stigma: a knowledge translation study in bipolar disorder. Int J Bipolar Disord. 2014;2:1.

146. Morrison PA. Using an adapted reflecting team approach to learn about mental health and illness with general nursing students: an Australian example. Int J Ment Health Nurs. 2009;18(1):18-25. https://doi.org/10.1111/ j.1447-0349.2008.00566.x.

147. Moxham L, Taylor E, Patterson C, Perlman D, Brighton R, Sumskis S, et al. Can a clinical placement influence stigma? An analysis of measures of social distance. Nurse Educ Today. 2016:44:170-4.

148. Muzyk AJ, Lentz K, Green C, Fuller S, May DB, Roukema L. Emphasizing Bloom's affective domain to reduce pharmacy Students' stigmatizing attitudes. Am J Pharm Educ. 2017:81(2):35. https://doi.org/10.5688/ajpe81235.

149. O'Connor K, Brennan D, O'Loughlin K, Wilson L, Pillay D, Clarke M, et al. Attitudes towards patients with mental illness in Irish medical students. Ir J Med Sci. 2013;182(4):679-85.

150. Omori A, Tateno A, Ideno T, Takahashi H, Kawashima Y, Takemura K, et al. Influence of contact with schizophrenia on implicit attitudes towards schizophrenia patients held by clinical residents. BMC Psychiatry. 2012;12:205.

151. O'Reilly CL, Bell JS, Chen TF. Consumer-led mental health education for pharmacy students. Am J Pharm Educ. 2010;74(9):167.

152. O'Reilly CL, Bell JS, Kelly PJ, Chen TF. Impact of mental health first aid training on pharmacy students' knowledge, attitudes and self-reported behaviour: a controlled trial. Aust New Zealand J Psychiatry. 2011:45(7):549-57.

153. Papish A, Kassam A, Modgill G, Vaz G, Zanussi L, Patten S. Reducing the stigma of mental illness in undergraduate medical education: a randomized controlled trial. BMC Med Educ. 2013;13:141.

154. Patten SB, Remillard A, Phillips L, Modgill G, Szeto AC, Kassam A, et al. Effectiveness of contact-based education for reducing mental illness-related stigma in pharmacy students. BMC Med Educ. 2012;12(1):120. https://doi. org/10.1186/1472-6920-12-120.

155. Poreddi $\mathrm{V}$, Thimmaiah R, Math SB. Attitudes toward people with mental illness among medical students. J Neurosci Rural Pract. 2015;6(3):349-54.

156. Romem P, Anson O, Kanat-Maymon Y, Moisa R. Reshaping students' attitudes toward individuals with mental illness through a clinical nursing clerkship. J Nurs Educ. 2008:47(9):396-402.

157. Shen Y, Dong H, Fan X, Zhang Z, Li L, Lv H, et al. What can the medical education do for eliminating stigma and discrimination associated with mental illness among future doctors? Effect of clerkship training on chinese 
students' attitudes. Int J Psychiatry Med. 2014;47(3):241-54. https://doi.org/1 0.2190/PM.47.3.e.

158. Stacey G, Baldwin V, Thompson BJ, Aubeeluck A. A focus group study exploring student nurse's experiences of an educational intervention focused on working with people with a diagnosis of personality disorder. J Psychiatr Ment Health Nurs. 2018:25(7):390-9.

159. Stuhlmiller C, Tolchard B. Understanding the impact of mental health placements on student nurses' attitudes towards mental illness. Nurse Educ Pract. 2019;34:25-30. https://doi.org/10.1016/j.nepr.2018.06.004.

160. Telles-Correia D, Gama Marques J, Gramaca J, Sampaio D. Stigma and attitudes towards psychiatric patients in Portuguese medical students. Acta Medica Port. 2015;28(6):715-9.

161. Treloar AJ. Effectiveness of education programs in changing clinicians' attitudes toward treating borderline personality disorder. Psychiatr Serv. 2009:60(8):1128-31.

162. Upshur C, Weinreb L. A survey of primary care provider attitudes and behaviors regarding treatment of adult depression: what changes after a collaborative care intervention? Primary Care Companion J Clin Psychiatry. 2008;10(3):182-6. https://doi.org/10.4088/PCC.v10n0301.

163. Wang P-W, Ko C-H, Chen C-S, Yang Y-H, Lin H-C, Cheng C-C, et al. Changes of explicit and implicit stigma in medical students during psychiatric clerkship. Acad Psychiatry. 2016;40(2):224-8. https://doi.org/10.1007/s40596015-0432-8.

164. Winkler P, Janouskova M, Kozeny J, Pasz J, Mlada K, Weissova A, et al. Short video interventions to reduce mental health stigma: a multi-Centre randomised controlled trial in nursing high schools. Soc Psychiatry Psychiatr Epidemiol. 2017;52(12):1549-57.

165. Clarke D, Usick R, Sanderson A, Giles-Smith L, Baker J. Emergency department staff attitudes towards mental health consumers: a literature review and thematic content analysis. Int J Ment Health Nurs. 2014;23(3): 273-84.

166. Rees N, Rapport F, Thomas G, John A, Snooks H. Perceptions of paramedic and emergency care workers of those who self harm: a systematic review of the quantitative literature. J Psychosom Res. 2014;77(6):449-56. https://doi. org/10.1016/j.jpsychores.2014.09.006.

167. Vistorte AOR, Ribeiro WS, Jaen D, Jorge MR, Evans-Lacko S, Mari JJ. Stigmatizing attitudes of primary care professionals towards people with mental disorders: a systematic review. Int J Psychiatry Med. 2018;53(4):317-38.

168. Petkari E, Masedo Gutierrez Al, Xavier M, Moreno Kustner B. The influence of clerkship on students' stigma towards mental illness: a meta-analysis. Med Educ. 2018;52(7):694-704.

169. Stubbs A. Reducing mental illness stigma in health care students and professionals: a review of the literature. Australas Psychiatry. 2014;22(6):579-84.

170. McHale J, Felton A. Self-harm: what's the problem? A literature review of the factors affecting attitudes towards self-harm. J Psychiatr Ment Health Nurs. 2010;17(8):732-40.

171. Saunders KEA, Hawton K, Fortune S, Farrell S. Attitudes and knowledge of clinical staff regarding people who self-harm: a systematic review. J Affect Disord. 2012;139(3):205-16.

\section{Publisher's Note}

Springer Nature remains neutral with regard to jurisdictional claims in published maps and institutional affiliations.

Ready to submit your research? Choose BMC and benefit from:

- fast, convenient online submission

- thorough peer review by experienced researchers in your field

- rapid publication on acceptance

- support for research data, including large and complex data types

- gold Open Access which fosters wider collaboration and increased citations

- maximum visibility for your research: over $100 \mathrm{M}$ website views per year

At BMC, research is always in progress.

Learn more biomedcentral.com/submissions 